\title{
Theorems on Existence and Global Dynamics for the Einstein Equations
}

\author{
Alan D. Rendall \\ Max-Planck-Institut für Gravitationsphysik \\ Am Mühlenberg 1 \\ 14424 Golm, Germany \\ email: rendall@aei.mpg.de \\ http://www . aei.mpg.de/〜rendall/rendall.html \\ Accepted on 28 September 2005 \\ Published on 18 October 2005 \\ Living Reviews in Relativity \\ Published by the \\ Max Planck Institute for Gravitational Physics \\ (Albert Einstein Institute) \\ Am Mühlenberg 1, 14424 Golm, Germany \\ ISSN 1433-8351
}

\begin{abstract}
This article is a guide to theorems on existence and global dynamics of solutions of the Einstein equations. It draws attention to open questions in the field. The local-in-time Cauchy problem, which is relatively well understood, is surveyed. Global results for solutions with various types of symmetry are discussed. A selection of results from Newtonian theory and special relativity that offer useful comparisons is presented. Treatments of global results in the case of small data and results on constructing spacetimes with prescribed singularity structure or late-time asymptotics are given. A conjectural picture of the asymptotic behaviour of general cosmological solutions of the Einstein equations is built up. Some miscellaneous topics connected with the main theme are collected in a separate section.
\end{abstract}

(c) Max Planck Society and the authors.

Further information on copyright is given at http://relativity.livingreviews .org/About/copyright.html

For permission to reproduce the article please contact livrev@aei.mpg.de. 


\section{How to cite this article}

Owing to the fact that a Living Reviews article can evolve over time, we recommend to cite the article as follows:

Alan D. Rendall,

"Theorems on Existence and Global Dynamics for the Einstein Equations",

Living Rev. Relativity, 8, (2005), 6. [Online Article]: cited [<date>],

http://www.livingreviews.org//rr-2005-6

The date given as $<$ date $>$ then uniquely identifies the version of the article you are referring to.

\section{Article Revisions}

Living Reviews supports two different ways to keep its articles up-to-date:

Fast-track revision A fast-track revision provides the author with the opportunity to add short notices of current research results, trends and developments, or important publications to the article. A fast-track revision is refereed by the responsible subject editor. If an article has undergone a fast-track revision, a summary of changes will be listed here.

Major update A major update will include substantial changes and additions and is subject to full external refereeing. It is published with a new publication number.

For detailed documentation of an article's evolution, please refer always to the history document of the article's online version at http://www. livingreviews.org/lrr-2005-6. 


\section{Contents}

1 Introduction $\quad 5$

2 Local Existence $\quad 7$

2.1 The constraints . . . . . . . . . . . . . . . . . . . . 7

2.2 The vacuum evolution equations . . . . . . . . . . . . . . . . 8

2.3 Questions of differentiability . . . . . . . . . . . . . . . . . . . . . . . . . . . . . . . . . . . . . . .

2.4 New techniques for rough solutions . . . . . . . . . . . . . . . . . . . 12

2.5 Matter fields . . . . . . . . . . . . . . . . . . . . . . . . . . . . . . . . . . . . . . . . . . . . . .

2.6 Free boundary problems . . . . . . . . . . . . . . . . . . . 13

3 Global Symmetric Solutions $\quad \mathbf{1 5}$

3.1 Stationary solutions . . . . . . . . . . . . . . . . . . . 15

3.2 Spatially homogeneous solutions . . . . . . . . . . . . . . . . . . . . . . . . . . . . . . . . . . .

3.3 Spherically symmetric asymptotically flat solutions . . . . . . . . . . . . . . . 18

3.4 Weak null singularities and Price's law . . . . . . . . . . . . . . . . . . . 20

3.5 Cylindrically symmetric solutions . . . . . . . . . . . . . . . . . 21

3.6 Spatially compact solutions . . . . . . . . . . . . . . . . . . . 21

4 Newtonian Theory and Special Relativity 2

4.1 Hydrodynamics . . . . . . . . . . . . . . . . . . . . . . . . 24

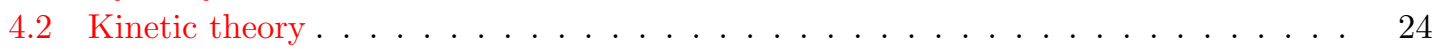

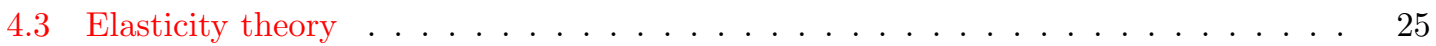

5 Global Existence for Small Data $\quad \mathbf{2 6}$

5.1 Stability of de Sitter space . . . . . . . . . . . . . . . . . . . . . . . . . . . . . . . . .

5.2 Stability of Minkowski space . . . . . . . . . . . . . . . . . . . . . 26

5.3 Stability of the (compactified) Milne model . . . . . . . . . . . . . . . . . . 27

5.4 Stability of the Bianchi type III form of flat spacetime . . . . . . . . . . . . . . . 27

6 Prescribed Asymptotics $\quad 29$

6.1 Isotropic singularities . . . . . . . . . . . . . . . . . . . . . . . . . . . . . . . . . . . . . . . . . . . . .

6.2 Fuchsian equations . . . . . . . . . . . . . . . . . . . . . . . . . . . . . . . . . . . . . . . . . .

6.3 Asymptotics for a phase of accelerated expansion . . . . . . . . . . . . . . . 30

7 Asymptotics of Expanding Cosmological Models $\quad 32$

7.1 Lessons from homogeneous solutions . . . . . . . . . . . . . . . . . . 32

7.2 Inhomogeneous solutions with $\Lambda=0 \ldots \ldots \ldots$. . . . . . . . . . 34

7.3 Homogeneous models with $\Lambda>0 \ldots \ldots \ldots \ldots$

7.4 Acceleration due to nonlinear scalar fields . . . . . . . . . . . . . . . . . . . . . . . . . . . . . . . . . . . . . . . .

7.5 Other models for cosmic acceleration . . . . . . . . . . . . . . . . . . . . . 37

7.6 Inhomogeneous spacetimes with accelerated expansion . . . . . . . . . . . 38

8 Structure of General Singularities $\quad \mathbf{4 0}$

8.1 Lessons from homogeneous solutions . . . . . . . . . . . . . . . . . . 40

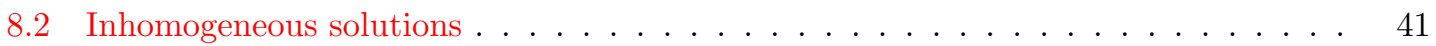

8.3 Formation of localized structure . . . . . . . . . . . . . . . . . . 41

8.4 Cosmic censorship in Gowdy spacetimes . . . . . . . . . . . . . . . . . . . 42 
9 Further Results $\quad 43$

9.1 Evolution of hyperboloidal data . . . . . . . . . . . . . . . . . . . . . . . . 43

9.2 The Newtonian limit . . . . . . . . . . . . . . . . . . . . . . . . . . . . . . . . . . . . . . . . . . . . . .

9.3 Newtonian cosmology . . . . . . . . . . . . . . . . . . . . . . 44

9.4 The characteristic initial value problem . . . . . . . . . . . . . . . . . . . 44

9.5 The initial boundary value problem . . . . . . . . . . . . . . . . . . . . . . . . . . . . . . . .

9.6 The geodesic hypothesis . . . . . . . . . . . . . . . . . . 45

10 Acknowledgements $\quad 46$

$\begin{array}{lr}\text { References } & 68\end{array}$ 


\section{Introduction}

Systems of partial differential equations are of central importance in physics. Only the simplest of these equations can be solved by explicit formulae. Those that cannot are commonly studied by means of approximations. There is, however, another approach that is complementary. This consists in determining the qualitative behaviour of solutions, without knowing them explicitly. The first step in doing this is to establish the existence of solutions under appropriate circumstances. Unfortunately, this is often hard, and obstructs the way to obtaining more interesting information. When partial differential equations are investigated with a view to applications, proving existence theorems should not become an end in itself. It is important to remember that, from a more general point of view, it is only a starting point.

The basic partial differential equations of general relativity are Einstein's equations. In general, they are coupled to other partial differential equations describing the matter content of spacetime. The Einstein equations are essentially hyperbolic in nature. In other words, the general properties of solutions are similar to those found for the wave equation. It follows that it is reasonable to try to determine a solution by initial data on a spacelike hypersurface. Thus the Cauchy problem is the natural context for existence theorems for the Einstein equations. The Einstein equations are also nonlinear. This means that there is a big difference between the local and global Cauchy problems. A solution evolving from regular data may develop singularities.

A special feature of the Einstein equations is that they are diffeomorphism invariant. If the equations are written down in an arbitrary coordinate system, then the solutions of these coordinate equations are not uniquely determined by initial data. Applying a diffeomorphism to one solution gives another solution. If this diffeomorphism is the identity on the chosen Cauchy surface up to first order then the data are left unchanged by this transformation. In order to obtain a system for which uniqueness in the Cauchy problem holds in the straightforward sense that it does for the wave equation, some coordinate or gauge fixing must be carried out.

Another special feature of the Einstein equations is that initial data cannot be prescribed freely. They must satisfy constraint equations. To prove the existence of a solution of the Einstein equations, it is first necessary to prove the existence of a solution of the constraints. The usual method of solving the constraints relies on the theory of elliptic equations.

The local existence theory of solutions of the Einstein equations is rather well understood. Section 2 points out some of the things that are not known. On the other hand, the problem of proving general global existence theorems for the Einstein equations is beyond the reach of the mathematics presently available. To make some progress, it is necessary to concentrate on simplified models. The most common simplifications are to look at solutions with various types of symmetry and solutions for small data. These two approaches are reviewed in Sections 3 and 5, respectively. A different approach is to prove the existence of solutions with a prescribed singularity structure or late-time asymptotics. This is discussed in Section 6. Section 9 collects some miscellaneous results that cannot easily be classified. Since insights about the properties of solutions of the Einstein equations can be obtained from the comparison with Newtonian theory and special relativity, relevant results from those areas are presented in Section 4.

The sections just listed are to some extent catalogues of known results, augmented with some suggestions as to how these could be extended in the future. Sections 7 and 8 complement this by looking ahead to see what the final answer to some interesting general questions might be. They are necessarily more speculative than the other sections but are rooted in the known results surveyed elsewhere in the article. Section 7 also summarizes various results on cosmological models with accelerated expansion.

The area of research reviewed in the following relies heavily on the theory of differential equations, particularly that of hyperbolic partial differential equations. For the benefit of readers with little background in differential equations, some general references that the author has found to be 
useful will be listed. A thorough introduction to ordinary differential equations is given in [170]. A lot of intuition for ordinary differential equations can be obtained from [186]. The article by Arnold and Ilyashenko [29] is full of information, in rather compressed form. A classic introductory text on partial differential equations, where hyperbolic equations are well represented, is [197]. Useful texts on hyperbolic equations, some of which explicitly deal with the Einstein equations, are [335, 202, 269, 239, 327, 198, 137].

An important aspect of existence theorems in general relativity that one should be aware of is their relation to the cosmic censorship hypothesis. This point of view was introduced in an influential paper by Moncrief and Eardley [248]. An extended discussion of the idea can be found in [114].

This article is descriptive in nature and equations have been kept to a minimum. A collection of relevant equations together with the background necessary to understand the notation can be found in [301].

Living Reviews in Relativity

http://www.livingreviews . org/lrr-2005-6 


\section{Local Existence}

In this section basic facts about local existence theorems for the Einstein equations are recalled. Since the theory is well developed and good accounts exist elsewhere (see for instance [148]), attention is focussed on remaining open questions known to the author. In particular, the questions of the minimal regularity required to get a well-posed problem and of free boundaries for fluid bodies are discussed.

\subsection{The constraints}

The unknowns in the constraint equations are the initial data for the Einstein equations. These consist of a three-dimensional manifold $S$, a Riemannian metric $h_{a b}$, and a symmetric tensor $k_{a b}$ on $S$, and initial data for any matter fields present. The equations are:

$$
\begin{aligned}
R-k_{a b} k^{a b}+\left(h^{a b} k_{a b}\right)^{2} & =16 \pi \rho, \\
\nabla^{a} k_{a b}-\nabla_{b}\left(h^{a c} k_{a c}\right) & =8 \pi j_{b} .
\end{aligned}
$$

Here $R$ is the scalar curvature of the metric $h_{a b}$, and $\rho$ and $j_{a}$ are projections of the energymomentum tensor. Assuming that the matter fields satisfy the dominant energy condition implies that $\rho \geq\left(j_{a} j^{a}\right)^{1 / 2}$. This means that the trivial procedure of making an arbitrary choice of $h_{a b}$ and $k_{a b}$ and defining $\rho$ and $j_{a}$ by Equations (1) is of no use for producing physically interesting solutions.

The usual method for solving the Equations (1) is the conformal method [95]. In this method parts of the data (the so-called free data) are chosen, and the constraints imply four elliptic equations for the remaining parts. The case that has been studied the most is the constant mean curvature (CMC) case, where $\operatorname{tr} k=h^{a b} k_{a b}$ is constant. In that case there is an important simplification. Three of the elliptic equations, which form a linear system, decouple from the remaining one. This last equation, which is nonlinear, but scalar, is called the Lichnerowicz equation. The heart of the existence theory for the constraints in the CMC case is the theory of the Lichnerowicz equation.

Solving an elliptic equation is a non-local problem and so boundary conditions or asymptotic conditions are important. For the constraints, the cases most frequently considered in the literature are that where $S$ is compact (so that no boundary conditions are needed) and that where the free data satisfy some asymptotic flatness conditions. In the CMC case the problem is well understood for both kinds of boundary conditions [76, 110, 187]. (Note that a significant mistake in the literature has been corrected in [244], section 4.) The other case that has been studied in detail is that of hyperboloidal data [9]. The kind of theorem that is obtained is that sufficiently differentiable free data, in some cases required to satisfy some global restrictions, can be completed in a unique way to a solution of the constraints. It should be noted in passing that in certain cases physically interesting free data may not be "sufficiently differentiable" in the sense it is meant here. One such case is mentioned at the end of Section 2.6. The usual kinds of differentiability conditions that are required in the study of the constraints involve the free data belonging to suitable Sobolev or Hölder spaces. Sobolev spaces have the advantage that they fit well with the theory of the evolution equations (compare the discussion in Section 2.2). The question of the minimal differentiability necessary to apply the conformal method has been studied in [243], where it was shown that the method works for metrics in the Sobolev space $H^{s}$ with $s>3 / 2$. It was also shown that each of these solutions can be approximated by a sequence of smooth solutions.

Usually it is not natural to prescribe the values of solutions of the Einstein equations on a finite boundary. There is, however, one case which naturally occurs in physical problems, namely that of the boundary of a black hole. Existence of solutions of the constraints appropriate for describing black holes has been proved by solving boundary value problems in [127] and [244]. 
In the non-CMC case our understanding is much more limited although some results have been obtained in recent years (see [191, 93] and references therein). It is an important open problem to extend these so that an overview is obtained comparable to that available in the CMC case. Progress on this could also lead to a better understanding of the question of whether a spacetime that admits a compact, or asymptotically flat, Cauchy surface also admits one of constant mean curvature. Up to now there have been only isolated examples that exhibit obstructions to the existence of CMC hypersurfaces [34]. Until very recently it was not known whether there were vacuum spacetimes with a compact Cauchy surface admitting no CMC hypersurfaces. In [117] it was shown using gluing techniques (see below) that spacetimes of this type do exist and this fact restricts the applicability of CMC foliations for defining a preferred time coordinate in cosmological spacetimes. Certain limitations of the conformal method in producing non-CMC initial data sets were exhibited in [193].

It would be interesting to know whether there is a useful concept of the most general physically reasonable solutions of the constraints representing regular initial configurations. Data of this kind should not themselves contain singularities. Thus it seems reasonable to suppose at least that the metric $h_{a b}$ is complete and that the length of $k_{a b}$, as measured using $h_{a b}$, is bounded. Does the existence of solutions of the constraints imply a restriction on the topology of $S$ or on the asymptotic geometry of the data? This question is largely open, and it seems that information is available only in the compact and asymptotically flat cases. In the case of compact $S$, where there is no asymptotic regime, there is known to be no topological restriction. In the asymptotically flat case there is also no topological restriction implied by the constraints beyond that implied by the condition of asymptotic flatness itself [349, 189]. This shows in particular that any manifold that is obtained by deleting a point from a compact manifold admits a solution of the constraints satisfying the minimal conditions demanded above. A starting point for going beyond this could be the study of data that are asymptotically homogeneous. For instance, the Schwarzschild solution contains interesting CMC hypersurfaces that are asymptotic to the metric product of a round 2 -sphere with the real line. More general data of this kind could be useful for the study of the dynamics of black hole interiors [292].

Recently techniques have been developed for gluing together solutions of the constraints (see [117] and references therein). Given two solutions of the constraints it is possible, under very general conditions, to cut a hole in each and connect the resulting pieces by a wormhole to get a new solution of the constraints. Depending on the variant of the method used, the geometry on the original pieces is changed by an arbitrarily small amount, or not at all. This gives a new flexibility in constructing solutions of the constraints with interesting properties.

To sum up, the conformal approach to solving the constraints, which has been the standard one up to now, is well understood in the compact, asymptotically flat and hyperboloidal cases under the constant mean curvature assumption, and only in these cases. For some other approaches see [35, 36, 353]. New techniques have been applied by Corvino [121] to prove the existence of regular solutions of the vacuum constraints on $\mathbf{R}^{3}$ that are Schwarzschild outside a compact set. The latter ideas have also flowed into the gluing constructions mentioned above.

\subsection{The vacuum evolution equations}

The main aspects of the local-in-time existence theory for the Einstein equations can be illustrated by restricting to smooth (i.e. infinitely differentiable) data for the vacuum Einstein equations. The generalizations to less smooth data and matter fields are discussed in Sections 2.3 and 2.5, respectively. In the vacuum case, the data are $h_{a b}$ and $k_{a b}$ on a three-dimensional manifold $S$, as discussed in Section 2.1. A solution corresponding to these data is given by a four-dimensional manifold $M$, a Lorentz metric $g_{\alpha \beta}$ on $M$, and an embedding of $S$ in $M$. Here, $g_{\alpha \beta}$ is supposed to be a solution of the vacuum Einstein equations, while $h_{a b}$ and $k_{a b}$ are the induced metric and

Living Reviews in Relativity

http://www . livingreviews . org//rr-2005-6 
second fundamental form of the embedding, respectively.

The basic local existence theorem says that, given smooth data for the vacuum Einstein equations, there exists a smooth solution of the equations which gives rise to these data [95]. Moreover, it can be assumed that the image of $S$ under the given embedding is a Cauchy surface for the metric $g_{\alpha \beta}$. The latter fact may be expressed loosely, identifying $S$ with its image, by the statement that $S$ is a Cauchy surface. A solution of the Einstein equations with given initial data having $S$ as a Cauchy surface is called a Cauchy development of those data. The existence theorem is local because it says nothing about the size of the solution obtained. A Cauchy development of given data has many open subsets that are also Cauchy developments of that data.

It is intuitively clear what it means for one Cauchy development to be an extension of another. The extension is called proper if it is strictly larger than the other development. A Cauchy development that has no proper extension is called maximal. The standard global uniqueness theorem for the Einstein equations uses the notion of the maximal development. It is due to Choquet-Bruhat and Geroch [91]. It says that the maximal development of any Cauchy data is unique up to a diffeomorphism that fixes the initial hypersurface. It is also possible to make a statement of Cauchy stability that says that, in an appropriate sense, the solution depends continuously on the initial data. Details on this can be found in [95].

A somewhat stronger form of the local existence theorem is to say that the solution exists on a uniform time interval in all of space. The meaning of this is not a priori clear, due to the lack of a preferred time coordinate in general relativity. The following is a formulation that is independent of coordinates. Let $p$ be a point of $S$. The temporal extent $T(p)$ of a development of data on $S$ is the supremum of the length of all causal curves in the development passing through $p$. In this way, a development defines a function $T$ on $S$. The development can be regarded as a solution that exists on a uniform time interval if $T$ is bounded below by a strictly positive constant. For compact $S$ this is a straightforward consequence of Cauchy stability. In the case of asymptotically flat data it is less trivial. In the case of the vacuum Einstein equations it is true, and in fact the function $T$ grows at least linearly as a function of spatial distance at infinity [110]. It should follow from the results of [211] that the constant of proportionality in the linear lower bound for $T$ can be chosen to be unity, but this does not seem to have been worked out explicitly.

When proving the above local existence and global uniqueness theorems it is necessary to use some coordinate or gauge conditions. At least no explicitly diffeomorphism-invariant proofs have been found up to now. Introducing these extra elements leads to a system of reduced equations, whose solutions are determined uniquely by initial data in the strict sense, and not just uniquely up to diffeomorphisms. When a solution of the reduced equations has been obtained, it must be checked that it is a solution of the original equations. This means checking that the constraints and gauge conditions propagate. There are many methods for reducing the equations. An overview of the possibilities may be found in [144]. See also [148].

\subsection{Questions of differentiability}

Solving the Cauchy problem for a system of partial differential equations involves specifying a set of initial data to be considered, and determining the differentiability properties of solutions. Thus, two regularity properties are involved - the differentiability of the allowed data, and that of the corresponding solutions. Normally, it is stated that for all data with a given regularity, solutions with a certain type of regularity are obtained. For instance, in Section 2.2 we chose both types of regularity to be "infinitely differentiable". The correspondence between the regularity of data and that of solutions is not a matter of free choice. It is determined by the equations themselves, and in general the possibilities are severely limited. A similar issue arises in the context of the Einstein constraints, where there is a correspondence between the regularity of free data and full data.

The kinds of regularity properties that can be dealt with in the Cauchy problem depend, of

Living Reviews in Relativity

http ://www . livingreviews . org/lrr-2005-6 
course, on the mathematical techniques available. When solving the Cauchy problem for the Einstein equations, it is necessary to deal at least with nonlinear systems of hyperbolic equations. (There may be other types of equations involved, but they will be ignored here.) For general nonlinear systems of hyperbolic equations the standard technique is the method of energy estimates. This method is closely connected with Sobolev spaces, which will now be discussed briefly.

Let $u$ be a real-valued function on $\mathbf{R}^{n}$. Let

$$
\|u\|_{s}=\left(\sum_{i=0}^{s} \int\left|D^{i} u\right|^{2}(x) d x\right)^{1 / 2}
$$

The space of functions for which this quantity is finite is the Sobolev space $H^{s}\left(\mathbf{R}^{n}\right)$. Here, $\left|D^{i} u\right|^{2}$ denotes the sum of the squares of all partial derivatives of $u$ of order $i$. Thus, the Sobolev space $H^{s}$ is the space of functions, all of whose partial derivatives up to order $s$ are square integrable. Similar spaces can be defined for vector valued functions by taking a sum of contributions from the separate components in the integral. It is also possible to define Sobolev spaces on any Riemannian manifold, using covariant derivatives. General information on this can be found in [30]. Consider now a solution $u$ of the wave equation in Minkowski space. Let $u(t)$ be the restriction of this function to a time slice. Then it is easy to compute that, provided $u$ is smooth and $u(t)$ has compact support for each $t$, the quantity $\|D u(t)\|_{s}^{2}+\left\|\partial_{t} u(t)\right\|_{s}^{2}$ is time independent for each $s$. For $s=0$ this is just the energy of a solution of the wave equation. For a general nonlinear hyperbolic system, the Sobolev norms are no longer time-independent. The constancy in time is replaced by certain inequalities. Due to the similarity to the energy for the wave equation, these are called energy estimates. They constitute the foundation of the theory of hyperbolic equations. It is because of these estimates that Sobolev spaces are natural spaces of initial data in the Cauchy problem for hyperbolic equations. The energy estimates ensure that a solution evolving from data belonging to a given Sobolev space on one spacelike hypersurface will induce data belonging to the same Sobolev space on later spacelike hypersurfaces. In other words, the property of belonging to a Sobolev space is propagated by the equations. Due to the locality properties of hyperbolic equations (existence of a finite domain of dependence), it is useful to introduce the spaces $H_{\text {loc }}^{s}$, which are defined by the condition that whenever the domain of integration is restricted to a compact set, the integral defining the space $H^{s}$ is finite.

In the end, the solution of the Cauchy problem should be a function that is differentiable enough so that all derivatives that occur in the equation exist in the usual (pointwise) sense. A square integrable function is in general defined only almost everywhere and the derivatives in the above formula must be interpreted as distributional derivatives. For this reason, a connection between Sobolev spaces and functions whose derivatives exist pointwise is required. This is provided by the Sobolev embedding theorem. This says that if a function $u$ on $\mathbf{R}^{n}$ belongs to the Sobolev space $H_{\text {loc }}^{s}$ and if $k<s-n / 2$, then there is a $k$ times continuously differentiable function that agrees with $u$ except on a set of measure zero.

In the existence and uniqueness theorems stated in Section 2.2, the assumptions on the initial data for the vacuum Einstein equations can be weakened to say that $h_{a b}$ should belong to $H_{\text {loc }}^{s}$ and $k_{a b}$ to $H_{\text {loc }}^{s-1}$. Then, provided $s$ is large enough, a solution is obtained that belongs to $H_{\text {loc }}^{s}$. In fact, its restriction to any spacelike hypersurface also belongs to $H_{\mathrm{loc}}^{s}$, a property that is $a$ priori stronger. The details of how large $s$ must be would be out of place here, since they involve examining the detailed structure of the energy estimates. However, there is a simple rule for computing the required value of $s$. The value of $s$ needed to obtain an existence theorem for the Einstein equations using energy estimates is that for which the Sobolev embedding theorem, applied to spatial slices, just ensures that the metric is continuously differentiable. Thus the requirement is that $s>n / 2+1=5 / 2$, since $n=3$. It follows that the smallest possible integer $s$ is three. Strangely enough, the standard methods only give uniqueness up to diffeomorphisms for

Living Reviews in Relativity

http://www. livingreviews.org//rr-2005-6 
$s \geq 4$. The reason is that in proving the uniqueness theorem a diffeomorphism must be carried out, which need not be smooth. This apparently leads to a loss of one derivative. In [10] local existence and uniqueness for the vacuum Einstein equations was proved using a gauge condition defined by elliptic equations for which this loss does not occur. In that case the gap of one derivative is eliminated. On the other hand, the occurrence of elliptic equations as part of the reduced Einstein equations with this gauge makes the result intrinsically global, and it is not clear whether it can be localized in space. Another interesting aspect of the main theorem of [10] is that it includes a continuation criterion for solutions. There exists a definition of Sobolev spaces for an arbitrary real number $s$, and hyperbolic equations can also be solved in the spaces with $s$ not an integer [334]. Presumably these techniques could be applied to prove local existence for the Einstein equations with $s$ any real number greater than $5 / 2$. In any case, the condition for local existence has been weakened to $s>2$ using other techniques, as discussed in Section 2.4.

Consider now $C^{\infty}$ initial data. Corresponding to these data there is a development of class $H^{s}$ for each $s$. It could conceivably be the case that the size of these developments shrinks with increasing $s$. In that case, their intersection might contain no open neighbourhood of the initial hypersurface, and no smooth development would be obtained. Fortunately, it is known that the $H^{s}$ developments cannot shrink with increasing $s$ [87], and so the existence of a $C^{\infty}$ solution is obtained for $C^{\infty}$ data. It appears that the $H^{s}$ spaces with $s$ sufficiently large are the only spaces containing the space of smooth functions for which it has been proved that the Einstein equations are locally solvable.

What is the motivation for considering regularity conditions other than the apparently very natural $C^{\infty}$ condition? One motivation concerns matter fields and will be discussed in Section 2.5. Another is the idea that assuming the existence of many derivatives that have no direct physical significance seems like an admission that the problem has not been fully understood. A further reason for considering low regularity solutions is connected to the possibility of extending a local existence result to a global one. If the proof of a local existence theorem is examined closely it is generally possible to give a continuation criterion. This is a statement that if a solution on a finite time interval is such that a certain quantity constructed from the solution is bounded on that interval, then the solution can be extended to a longer time interval. (In applying this to the Einstein equations we need to worry about introducing an appropriate time coordinate.) If it can be shown that the relevant quantity is bounded on any finite time interval where a solution exists, then global existence follows. It suffices to consider the maximal interval on which a solution is defined, and obtain a contradiction if that interval is finite. This description is a little vague, but contains the essence of a type of argument that is often used in global existence proofs. The problem in putting it into practice is that often the quantity whose boundedness has to be checked contains many derivatives, and is therefore difficult to control. If the continuation criterion can be improved by reducing the number of derivatives required, then this can be a significant step toward a global result. Reducing the number of derivatives in the continuation criterion is closely related to reducing the number of derivatives of the data required for a local existence proof.

A striking example is provided by the work of Klainerman and Machedon [210] on the YangMills equations in Minkowski space. Global existence in this case was first proved by Eardley and Moncrief [135], assuming initial data of sufficiently high differentiability. Klainerman and Machedon gave a new proof of this, which, though technically complicated, is based on a conceptually simple idea. They prove a local existence theorem for data of finite energy. Since energy is conserved this immediately proves global existence. In this case finite energy corresponds to the Sobolev space $H^{1}$ for the gauge potential. Of course, a result of this kind cannot be expected for the Einstein equations, since spacetime singularities do sometimes develop from regular initial data. However, some weaker analogue of the result could exist. 


\subsection{New techniques for rough solutions}

Recently, new mathematical techniques have been developed to lower the threshold of differentiability required to obtain local existence for quasilinear wave equations in general and the Einstein equations in particular. Some aspects of this development will now be discussed following [209, 214]. A central aspect is that of Strichartz inequalities. These allow one to go beyond the theory based on $L^{2}$ spaces and use Sobolev spaces based on the Lebesgue $L^{p}$ spaces for $p \neq 2$. The classical approach to deriving Strichartz estimates is based on the Fourier transform and applies to flat space. The new ideas allow the use of the Fourier transform to be limited to that of Littlewood-Paley theory and facilitate generalizations to curved space.

The idea of Littlewood-Paley theory is as follows (see [1] for a good exposition of this). Suppose that we want to describe the regularity of a function (or, more generally, a tempered distribution) $u$ on $\mathbf{R}^{n}$. Differentiability properties of $u$ correspond, roughly speaking, to fall-off properties of its Fourier transform $\hat{u}$. This is because the Fourier transform converts differentiation into multiplication. The Fourier transform is decomposed as $\hat{u}=\sum \phi_{i} \hat{u}$, where $\phi_{i}$ is a dyadic partition of unity. The statement that it is dyadic means that all the $\phi_{i}$ except one are obtained from each other by scaling the argument by a factor which is a power of two. Transforming back we get the decomposition $u=\sum u_{i}$, where $u_{i}$ is the inverse Fourier transform of $\phi_{i} \hat{u}$. The component $u_{i}$ of $u$ contains only frequencies of the order $2^{i}$. In studying rough solutions of the Einstein equations, the Littlewood-Paley decomposition is applied to the metric itself. The high frequencies are discarded to obtain a smoothed metric which plays an important role in the arguments.

Another important element of the proofs is to rescale the solution by a factor depending on the cut-off $\lambda$ applied in the Littlewood-Paley decomposition. Proving the desired estimates then comes down to proving the existence of the rescaled solutions on a time interval depending on $\lambda$ in a particular way. The rescaled data are small in some sense and so a connection is established to the question of long-time existence of solutions of the Einstein equations for small initial data. In this way, techniques from the work of Christodoulou and Klainerman on the stability of Minkowski space (see Section 5.2) are brought in.

What is finally proved? In general, there is a close connection between proving local existence for data in a certain space and showing that the time of existence of smooth solutions depends only on the norm of the data in the given space. Klainerman and Rodnianski [214] demonstrate that the time of existence of solutions of the reduced Einstein equations in harmonic coordinates depends only on the $H^{2+\epsilon}$ norm of the initial data for any $\epsilon>0$. Combining this with the results of [243] gives an existence result in the same space. It is of interest to try to push the existence theorem to the limiting case $\epsilon=0$ or even to the slightly weaker assumption on the data that the curvature is square integrable. This $L^{2}$ curvature conjecture (local existence in this setting) is extremely difficult but interesting progress has been made by Klainerman and Rodnianski in [215] where the structure of null hypersurfaces was analysed under very weak hypotheses. For this purpose the authors developed an invariant form of Littlewood-Paley theory [216]. This uses the asymptotics of solutions of the heat equation on a manifold and is coordinate-independent.

The techniques discussed in this section, which have been stimulated by the desire to understand the Einstein equations, are also helpful in understanding other nonlinear wave equations. Thus, this is an example where information can flow from general relativity to the theory of partial differential equations.

It may be that the technique of using parabolic equations as a tool to better understand hyperbolic equations can be carried much further. In [333] Tao presents ideas how the harmonic map heat flow could be used to define a high quality gauge for the study of wave maps.

Living Reviews in Relativity

http://www. livingreviews.org/lrr-2005-6 


\subsection{Matter fields}

Analogues of the results for the vacuum Einstein equations given in Section 2.2 are known for the Einstein equations coupled to many types of matter model. These include perfect fluids, elasticity theory [43], kinetic theory, scalar fields, Maxwell fields, Yang-Mills fields, and combinations of these. An important restriction is that the general results for perfect fluids and elasticity apply only to situations where the energy density is uniformly bounded away from zero on the region of interest. In particular, they do not apply to cases representing material bodies surrounded by vacuum. In cases where the energy density, while everywhere positive, tends to zero at infinity, a local solution is known to exist, but it is not clear whether a local existence theorem can be obtained that is uniform in time. In cases where the fluid has a sharp boundary, ignoring the boundary leads to solutions of the Einstein-Euler equations with low differentiability (cf. Section 2.3), while taking it into account explicitly leads to a free boundary problem. This will be discussed in more detail in Section 2.6. In the case of kinetic or field theoretic matter models it makes no difference whether the energy density vanishes somewhere or not.

\subsection{Free boundary problems}

In applying general relativity one would like to have solutions of the Einstein-matter equations modelling material bodies. As will be discussed in Section 3.1 there are solutions available for describing equilibrium situations. However, dynamical situations require solving a free boundary problem if the body is to be made of fluid or an elastic solid. We will now discuss the few results which are known on this subject. For a spherically symmetric self-gravitating fluid body in general relativity, a local-in-time existence theorem was proved in [206]. This concerned the case in which the density of the fluid at the boundary is non-zero. In [285] a local existence theorem was proved for certain equations of state with vanishing boundary density. These solutions need not have any symmetry but they are very special in other ways. In particular, they do not include small perturbations of the stationary solutions discussed in Section 3.1. There is no general result on this problem up to now.

Remarkably, the free boundary problem for a fluid body is also poorly understood in classical physics. There is a result for a viscous fluid [319], but in the case of a perfect fluid the problem was wide open until recently. A major step forward was taken by $\mathrm{Wu}$ [352], who obtained a result for a fluid that is incompressible and irrotational. There is a good physical reason why local existence for a fluid with a free boundary might fail. This is the Rayleigh-Taylor instability which involves perturbations of fluid interfaces that grow with unbounded exponential rates (cf. the discussion in [41]). It turns out that in the case considered by Wu this instability does not cause problems, and there is no reason to expect that a self-gravitating compressible fluid with rotation in general relativity with a free boundary cannot also be described by a well-posed free boundary value problem. For the generalization of the problem considered by $\mathrm{Wu}$ to the case of a fluid with rotation, Christodoulou and Lindblad [109] have obtained estimates that look as if they should be enough to obtain an existence theorem. Strangely, it proved very difficult to complete the argument. This point deserves some further comment. In many problems the heart of an existence proof is obtaining suitable estimates. Then more or less standard approximation techniques can be used to obtain the desired conclusion (for a discussion of this see [148], Section 3.1). In the problem studied in [109] it is an appropriate approximation method that is missing. More recently Lindblad was able to obtain an existence result using a different approach involving the Nash-Moser theorem and a detailed analysis of the linearized system about a given solution. He treated the incompressible case in [231] while in the case of a compressible fluid with non-vanishing boundary density the linearized analysis has been carried out [230] .

One of the problems in tackling the initial value problem for a dynamical fluid body is that the boundary is moving. It would be very convenient to use Lagrangian coordinates, since in those 
coordinates the boundary is fixed. Unfortunately, it is not at all obvious that the Euler equations in Lagrangian coordinates have a well-posed initial value problem, even in the absence of a boundary. It was, however, recently shown by Friedrich [145] that it is possible to treat the Cauchy problem for fluids in general relativity in Lagrangian coordinates.

In the case of a fluid with non-vanishing boundary density it is not only the evolution equations that cause problems. It is already difficult to construct suitable solutions of the constraints. A theorem on this has recently been obtained by Dain and Nagy [128]. There remains an undesirable technical restriction, but the theorem nevertheless provides a very general class of physically interesting initial data for a self-gravitating fluid body in general relativity.

Living Reviews in Relativity

http://www. livingreviews.org/lrr-2005-6 


\section{Global Symmetric Solutions}

An obvious procedure to obtain special cases of the general global existence problem for the Einstein equations that are amenable to attack is to make symmetry assumptions. In this section, we discuss the results obtained for various symmetry classes defined by different choices of number and character of Killing vectors.

The existence of a timelike Killing vector is a strong restriction on solutions of the Einstein equations. It corresponds physically to an equilibrium situation. This case is considered in Section 3.1. A null Killing vector also represents a strong restriction. It seems to fit badly with the Cauchy problem and is thus far from the discussion in this paper. This case will not be discussed further here. There remains the case where all Killing vectors are spacelike. The classes of solutions can be distinguished by the dimension of the group orbits. The case where the orbits are three-dimensional is that of spatially homogeneous spacetimes, discussed in Section 3.2. If the group orbits are two-dimensional there are important classes of solutions where the group is threeor two-dimensional. The case of a three-dimensional group includes asymptotically flat spherically symmetric solutions, discussed in Sections 3.3 and 3.4. It also includes spatially compact spacetimes with spherical, plane and hyperbolic symmetry, for which results are included in Section 3.6. That section also covers spatially compact solutions with a two-dimensional isometry group. Most results concern the situation where the Killing vectors have no zeroes. This means that when the spacetime is quotiented by the symmetry group in order to get an effective lower-dimensional system, no singularities occur in the reduced equations of motion. This is a big advantage for analytical work. The assumption of exactly two spacelike Killing vectors is not compatible with asymptotic flatness. It does allow asymptotic flatness in all directions except one and this is the subject of Section 3.5.

A single Killing vector without fixed points gives rise to an interesting class of cosmological models. Because of the perceived closeness to solutions without any Killing vectors, the results on these spacetimes have been placed in Section 5.4. Spacetimes with one fixed-point free Killing vector which are partly asymptotically flat are mentioned briefly in Section 3.5. Axially symmetric asymptotically flat spacetimes have one spacelike Killing vector with zeroes on the axis. It seems that no analytical advantage over the general case has been found for these spacetimes and so there are no results to report.

\subsection{Stationary solutions}

Many of the results on global solutions of the Einstein equations involve considering classes of spacetimes with Killing vectors. A particularly simple case is that of a timelike Killing vector, i.e. the case of stationary spacetimes. In the vacuum case there are very few solutions satisfying physically reasonable boundary conditions. This is related to no hair theorems for black holes and lies outside the scope of this review. More information on the topic can be found in the book of Heusler [181] and in his Living Review [182] (see also [55] where the stability of the Kerr metric is discussed). Anderson $[3,4]$ has proved uniqueness theorems for stationary vacuum spacetimes under very weak assumptions. The case of phenomenological matter models has been reviewed in [298]. The account given there will be updated in the following.

The area of stationary solutions of the Einstein equations coupled to field theoretic matter models has been active in recent years as a consequence of the discovery by Bartnik and McKinnon [37] of a discrete family of regular, static, spherically symmetric solutions of the Einstein-Yang-Mills equations with gauge group $S U(2)$. The equations to be solved are ordinary differential equations, and in [37] they were solved numerically by a shooting method. The first existence proof for a solution of this kind is due to Smoller, Wasserman, Yau, and McLeod [324] and involves an arduous qualitative analysis of the differential equations. The work on the Bartnik-McKinnon solutions, 
including the existence theorems, has been extended in many directions. Recently, static solutions of the Einstein-Yang-Mills equations that are not spherically symmetric were discovered numerically [217]. It is a challenge to prove the existence of solutions of this kind. Now the ordinary differential equations of the previously known case are replaced by elliptic equations. Moreover, the solutions appear to still be discrete, so that a simple perturbation argument starting from the spherical case does not seem feasible. In another development, it was shown that a linearized analysis indicates the existence of stationary non-static solutions [71]. It would be desirable to study the question of linearization stability in this case, which, if the answer were favourable, would give an existence proof for solutions of this kind. It has, however, been argued that solutions of this kind should not exist [343].

Now we return to phenomenological matter models, starting with the case of spherically symmetric static solutions. Basic existence theorems for this case have been proved for perfect fluids [307], collisionless matter [279, 271], and elastic bodies [265]. All these theorems demonstrate the existence of solutions that are everywhere smooth and exist globally as functions of area radius for a general class of constitutive relations. The physically significant question of the finiteness of the mass of these configurations was only answered in these papers under restricted circumstances. For instance, in the case of perfect fluids and collisionless matter, solutions were constructed by perturbing about the Newtonian case. Solutions for an elastic body were obtained by perturbing about the case of isotropic pressure, which is equivalent to a fluid. Further progress on the question of the finiteness of the mass of the solutions was made in the case of a fluid by Makino [240], who gave a rather general criterion on the equation of state ensuring the finiteness of the radius. Further information on this issue was obtained in [174] using dynamical systems methods. Makino's criterion was generalized to kinetic theory in [281]. This resulted in existence proofs for various models that have been considered in galactic dynamics and which had previously been constructed numerically (cf. [58, 320] for an account of these models in the non-relativistic and relativistic cases, respectively). In the non-relativistic case dynamical systems methods were applied to the case of collisionless matter in [173]. Most of the work quoted up to now refers to solutions where the support of the density is a ball. For matter with anisotropic pressure the support may also be a shell, i.e. the region bounded by two concentric spheres. The existence of static shells in the case of the Einstein-Vlasov equations was proved in [274].

In the case of self-gravitating Newtonian spherically symmetric configurations of collisionless matter, it can be proved that the phase space density of particles depends only on the energy of the particle and the modulus of its angular momentum [38]. This is known as Jeans' theorem. It was already shown in [271] that the naive generalization of this to the general relativistic case does not hold if a black hole is present. Recently, counterexamples to the generalization of Jeans' theorem to the relativistic case, which are not dependent on a black hole, were constructed by Schaeffer [318]. It remains to be seen whether there might be a natural modification of the formulation that would lead to a true statement.

For a perfect fluid there are results stating that a static solution is necessarily spherically symmetric [234]. They still require a restriction on the equation of state, which it would be desirable to remove. A similar result is not to be expected in the case of other matter models, although as yet no examples of non-spherical static solutions are available. In the Newtonian case examples have been constructed by Rein [275]. (In that case static solutions are defined to be those in which the particle current vanishes.) For a fluid there is an existence theorem for solutions that are stationary but not static (models for rotating stars) [172]. At present there are no corresponding theorems for collisionless matter or elastic bodies. In [275], stationary, non-static configurations of collisionless matter were constructed in the Newtonian case.

Two obvious characteristics of a spherically symmetric static solution of the Einstein-Euler equations that has a non-zero density only in a bounded spatial region are its radius $R$ and its total mass $M$. For a given equation of state there is a one-parameter family of solutions. These

Living Reviews in Relativity

http://www. livingreviews .org/lrr-2005-6 
trace out a curve in the $(M, R)$ plane. In the physics literature, pictures of this curve indicate that it spirals in on a certain point in the limit of large density. The occurrence of such a spiral and its precise asymptotic form have been proved rigorously by Makino [241] for a particular choice of equation of state. An approach to these spirals which leads to a better conceptual understanding can be found in [174].

The existence of cylindrically symmetric static solutions of the Einstein-Euler system has been proved in [56]. For some remarks on the question of stability of spherically symmetric solutions see Section 4.1.

\subsection{Spatially homogeneous solutions}

A solution of the Einstein equations is called spatially homogeneous if there exists a group of symmetries with three-dimensional spacelike orbits. In this case there are at least three linearly independent spacelike Killing vector fields. For most matter models the field equations reduce to ordinary differential equations. (Kinetic matter leads to an integro-differential equation.) The most important results in this area have been reviewed in the book [344] edited by Wainwright and Ellis (see, in particular, Part Two of the book). There remain a host of interesting and accessible open questions. The spatially homogeneous solutions have the advantage that it is not necessary to stop at just existence theorems; information on the global qualitative behaviour of solutions can also be obtained.

An important question that was open for a long time concerns the mixmaster model, as discussed in [296]. This is a class of spatially homogeneous solutions of the vacuum Einstein equations, which are invariant under the group $S U(2)$. A special subclass of these $S U(2)$-invariant solutions, the (parameter-dependent) Taub-NUT solution, is known explicitly in terms of elementary functions. The Taub-NUT solution has a simple initial singularity which is in fact a Cauchy horizon. All other vacuum solutions admitting a transitive action of $S U(2)$ on spacelike hypersurfaces (Bianchi type IX solutions) will be called generic in the present discussion. These generic Bianchi IX solutions (which might be said to constitute the mixmaster solution proper) have been believed for a long time to have singularities that are oscillatory in nature where some curvature invariant blows up. This belief was based on a combination of heuristic considerations and numerical calculations. Although these together do make a persuasive case for the accepted picture, until recently there were no mathematical proofs of these features of the mixmaster model available. This has now changed. First, a proof of curvature blow-up and oscillatory behaviour for a simpler model (a solution of the Einstein-Maxwell equations) which shares many qualitative features with the mixmaster model, was obtained by Weaver [347]. In the much more difficult case of the mixmaster model itself, corresponding results were obtained by Ringström [312]. Later he extended this in several directions in [311]. In that paper more detailed information was obtained concerning the asymptotics and an attractor for the evolution was identified. It was shown that generic solutions of Bianchi type IX with a perfect fluid whose equation of state is $p=(\gamma-1) \rho$ with $1 \leq \gamma<2$ are approximated near the singularity by vacuum solutions. The case of a stiff fluid $(\gamma=2)$ which has a different asymptotic behaviour was analysed completely for all models of Bianchi class A, a class which includes Bianchi type IX.

Ringström's analysis of the mixmaster model is potentially of great significance for the mathematical understanding of singularities of the Einstein equations in general. Thus, its significance goes far beyond the spatially homogeneous case. According to extensive investigations of Belinskii, Khalatnikov, and Lifshitz (see [226, 47, 48] and references therein), the mixmaster model should provide an approximate description for the general behaviour of solutions of the Einstein equations near singularities. This should apply to many matter models as well as to the vacuum equations. The work of Belinskii, Khalatnikov, and Lifshitz (BKL) is hard to understand and it is particularly difficult to find a precise mathematical formulation of their conclusions. This has caused many 
people to remain sceptical about the validity of the BKL picture. Nevertheless, it seems that nothing has ever been found to indicate any significant flaws in the final version. As long as the mixmaster model itself was not understood, this represented a fundamental obstacle to progress on understanding the BKL picture mathematically. The removal of this barrier opens up an avenue to progress on this issue. The BKL picture is discussed in more detail in Section 8.

Some recent and qualitatively new results concerning the asymptotic behaviour of spatially homogeneous solutions of the Einstein-matter equations, both close to the initial singularity and in a phase of unlimited expansion (and with various matter models), can be found in [308, 309, 302, 345, 259, 184, 180]. These show in particular that the dynamics can depend sensitively on the form of matter chosen. (Note that these results are consistent with the BKL picture.) The dynamics of indefinitely expanding cosmological models is discussed further in Section 7.

\subsection{Spherically symmetric asymptotically flat solutions}

The most extensive results on global inhomogeneous solutions of the Einstein equations obtained up to now concern spherically symmetric solutions of the Einstein equations coupled to a massless scalar field with asymptotically flat initial data. In a series of papers, Christodoulou [97, 96, 99, $98,100,101,102,106]$ has proved a variety of deep results on the global structure of these solutions. Particularly notable are his proofs that naked singularities can develop from regular initial data [102] and that this phenomenon is unstable with respect to perturbations of the data [106]. In related work, Christodoulou [103, 104, 105] has studied global spherically symmetric solutions of the Einstein equations coupled to a fluid with a special equation of state (the so-called two-phase model). Generalization of the results of [97] to the case of a nonlinear scalar field and to the Maxwell-Higgs system have been given by Chae [81, 82].

The rigorous investigation of the spherically symmetric collapse of collisionless matter in general relativity was initiated by Rein and the author [278], who showed that the evolution of small initial data leads to geodesically complete spacetimes where the density and curvature fall off at large times. Later, it was shown [282] that independent of the size of the initial data the first singularity, if there is one at all, must occur at the centre of symmetry. This result uses a time coordinate of Schwarzschild type; an analogous result for a maximal time coordinate was proved in [297]. The generalization of these results to the case of charged matter has been investigated in [261] and [260]. The question of what happens in the collapse of uncharged collisionless matter for general large initial data could not yet be answered by analytical techniques. In [283], numerical methods were applied to try to make some progress in this direction. The results are discussed below.

Some of the results of Christodoulou have been extended to much more general spacetimes by Dafermos [124]. In this work there are two basic assumptions. The first is the existence of at least one trapped surface in the spacetime under consideration. The second is that the matter content is well behaved in a certain sense which means intuitively that it does not form singularities outside black hole regions. Under these circumstances conclusions can be drawn on the global structure of the spacetime. It contains a black hole with a complete null infinity. It has been shown that collisionless matter has the desired property [125]. It also holds for certain nonlinear scalar fields and this has led to valuable insights in the discussion of the formation of naked singularities in a class of models motivated by string theory [179, 123].

Despite the range and diversity of the results obtained by Christodoulou on the spherical collapse of a scalar field, they do not encompass some of the most interesting phenomena that have been observed numerically. These are related to the issue of critical collapse. For sufficiently small data the field disperses. For sufficiently large data a black hole is formed. The question is what happens in between. This can be investigated by examining a one-parameter family of initial data interpolating between the two cases. It was found by Choptuik [86] that there is a critical value of the parameter below which dispersion takes place and above which a black hole is formed, and that

Living Reviews in Relativity

http://www. livingreviews.org/lrr-2005-6 
the mass of the black hole approaches zero as the critical parameter value is approached. This gave rise to a large literature in which the spherical collapse of different kinds of matter was computed numerically and various qualitative features were determined. For reviews of this see $[162,163]$. In the calculations of [283] for collisionless matter, it was found that in the situations considered the black hole mass tended to a strictly positive limit as the critical parameter was approached from above. These results were confirmed and extended by Olabarrieta and Choptuik [264]. There are no rigorous mathematical results available on the issue of a mass gap for either a scalar field or collisionless matter, and it is an outstanding challenge for mathematical relativists to change this situation.

Another aspect of Choptuik's results is the occurrence of a discretely self-similar solution. It would seem hard to prove the existence of a solution of this kind analytically. For other types of matter, such as a perfect fluid with linear equation of state, the critical solution is continuously self-similar and this looks more tractable. The problem reduces to solving a system of singular ordinary differential equations subject to certain boundary conditions. This problem was solved in [102] for the case where the matter model is given by a massless scalar field, but the solutions produced there, which are continuously self-similar, cannot include the Choptuik critical solution. Bizoń and Wasserman [62] studied the corresponding problem for the Einstein equations coupled to a wave map with target $S U(2)$. They proved the existence of continuously self-similar solutions including one which, according the results of numerical calculations, appears to play the role of critical solution in collapse. Another case where the question of the existence of the critical solution seems to be a problem that could possibly be solved in the near future is that of a perfect fluid. A good starting point for this is the work of Goliath, Nilsson, and Uggla [158, 159]. These authors gave a formulation of the problem in terms of dynamical systems and were able to determine certain qualitative features of the solutions (see also [77, 78]).

A possible strategy for learning more about critical collapse, pursued by Bizoń and collaborators, is to study model problems in flat space that exhibit features similar to those observed numerically in the case of the Einstein equations. Until now, only models showing continuous self-similarity have been found. These include wave maps in various dimensions and the YangMills equations in spacetimes of dimension greater than four. As mentioned in Section 2.3, it is known that in four dimensions there exist global smooth solutions of the Yang-Mills equations corresponding to rather general initial data $[135,210]$. In dimensions greater than five it is known that there exist solutions that develop singularities in finite time. This follows from the existence of continuously self-similar solutions [61]. Numerical evidence indicates that this type of blow-up is stable, i.e. occurs for an open set of initial data. The numerical work also indicates that there is a critical self-similar solution separating this kind of blow-up from dispersion. The spacetime dimension five is critical for Yang-Mills theory. Apparently singularities form, but in a different way from what happens in dimension six. There is as yet no rigorous proof of blow-up in five dimensions.

The various features of Yang-Mills theory just mentioned are mirrored in two dimensions less by wave maps with values in spheres [60]. In four dimensions, blow-up is known while in three dimensions there appears (numerically) to be a kind of blow-up similar to that found for YangMills in dimension five. There is no rigorous proof of blow-up. What is seen numerically is that the collapse takes place by scaling within a one-parameter family of static solutions. The case of wave maps is the most favourable known model problem for proving theorems about critical phenomena associated to singularity formation. The existence of a solution having the properties expected of the critical solution for wave maps in four dimensions has been proved in [59]. Some rigorous support for the numerical findings in three dimensions has been given by work of Struwe [328]. He showed, among other things, that if there is blow-up in finite time it must take place in a way resembling that observed in the numerical calculations.

Self-similar solutions are characteristic of what is called Type II critical collapse. In Type I 
collapse an analogous role is played by static solutions and quite a bit is known about the existence of these. For instance, in the case of the Einstein-Yang-Mills equations, it is one of the BartnikMcKinnon solutions mentioned in Section 3.1 which does this. In the case of collisionless matter the results of [264] show that at least in some cases critical collapse is mediated by a static solution in the form of a shell. There are existence results for shells of this kind [274] although no connection has yet been made between those shells whose existence has been proved and those which have been observed numerically in critical collapse calculations. Note that Martín-García and Gundlach [242] have presented a (partially numerical) construction of self-similar solutions of the Einstein-Vlasov system.

\subsection{Weak null singularities and Price's law}

The results of this section concern spherically symmetric solutions but in order to explain their significance they need to be presented in context. A non-rotating uncharged black hole is represented by the Schwarzschild solution, which contains a singularity. At this singularity the Kretschmann scalar $R_{\alpha \beta \gamma \delta} R^{\alpha \beta \gamma \delta}$ blows up uniformly and this represents an obstruction to extending the spacetime through the singularity, at least in a $C^{2}$ manner.

A rotating uncharged black hole is represented by the Kerr solution in which the Schwarzschild singularity is replaced by a Cauchy horizon. This horizon marks a pathology of the global causal structure of the solution but locally the geometry can be extended smoothly through it. A similar situation is found in the non-rotating charged black hole which is represented by the ReissnerNordström solution. These facts are worrying since they suggest that black holes may generally lead to causal pathologies. The rotating case is the more physically interesting one, but the charged case is a valuable model problem for the rotating case. Spherical symmetry leads to immense technical simplifications and so only that case will be discussed here. It is the only one where theorems on global existence and qualitative behaviour relevant to this problem are available.

It was early suggested that the Cauchy horizon of the Reissner-Nordström solution should be unstable and that a generic perturbation of the initial data would lead to its being replaced by a Schwarzschild-like singularity. This scenario turned out to be oversimplified. An alternative was suggested by Poisson and Israel [267]. In their picture a generic perturbation of the Reissner-Nordström data leads to the Cauchy horizon being replaced by what they call a weak null singularity. At this singularity the curvature blows up, but the metric can be extended through the singularity in a way which is continuous and non-degenerate. In this situation it is possible to make sense of the causal character of the singularity which turns out to be null. Furthermore, an important invariant, the Hawking mass, blows up at the singularity, a phenomenon known as mass inflation. All these conclusions were based on heuristic arguments which were later backed up by numerical results [185].

A mathematical understanding of these effects came with the work of Dafermos [122]. He showed how, starting from a characteristic initial value problem with data given on two null hypersurfaces, one of which is the event horizon, it is possible to prove that a weak null singularity forms and that there is mass inflation. He uses a model with an uncharged scalar field and a static charge and works entirely inside the black hole region.

Ideally one would wish to start with regular data on a standard Cauchy surface and control both formation of the black hole and the evolution in its interior. This requires using some kind of charged matter, e.g., a charged scalar field. This is what was done numerically in [185]. Analytically it remains out of reach at the moment.

In the original heuristic arguments it is important to make statements about the behaviour of the solution outside the black hole and what behaviour on the horizon results. Here there are classical heuristic results of Price [268] for a scalar field on a black hole background. He states that the scalar field falls off in a certain way along the horizon. Let us call this Price's law. Now

Living Reviews in Relativity

http://www. livingreviews.org/lrr-2005-6 
a form of Price's law and its analogue for the coupled spherically symmetric Einstein-scalar field system have been proved by Dafermos and Rodnianski [126]. Thus we have come a long way towards an understanding of the problem discussed here. This has required the development of new mathematical techniques and these may one day turn out to be of importance in understanding the nonlinear stability of black holes.

\subsection{Cylindrically symmetric solutions}

Solutions of the Einstein equations with cylindrical symmetry that are asymptotically flat in all directions allowed by the symmetry represent an interesting variation on asymptotic flatness. There are two Killing vectors, one translational (without fixed points) and one rotational (with fixed points on the axis). Since black holes are apparently incompatible with this symmetry, one may hope to prove geodesic completeness of solutions under appropriate assumptions. (It would be interesting to have a theorem making the statement about black holes precise.) A proof of geodesic completeness has been achieved for the Einstein vacuum equations and for the source-free EinsteinMaxwell equations in [51], building on global existence theorems for wave maps [112, 111]. For a quite different point of view on this question involving integrable systems see [351]. A recent paper of Hauser and Ernst [171] also appears to be related to this question. However, due to the great length of this text and its reliance on many concepts unfamiliar to this author, no further useful comments on the subject can be made here.

Solutions of the Einstein-Vlasov system with cylindrical symmetry have been studied by Fjällborg [140]. He shows global existence provided certain conditions are satisfied near the axis.

Cylindrical symmetry can be generalized by abandoning the rotational Killing vector while maintaining the translational one. This sitation does not seem to have been studied in the literature. It may be that results on solutions with approximate cylindrical symmetry may be obtained using the work of Krieger [218] on wave maps.

\subsection{Spatially compact solutions}

In the context of spatially compact spacetimes it is first necessary to ask what kind of global statements are to be expected. In a situation where the model expands indefinitely it is natural to pose the question whether the spacetime is causally geodesically complete towards the future. In a situation where the model develops a singularity either in the past or in the future one can ask what the qualitative nature of the singularity is. It is very difficult to prove results of this kind. As a first step one may prove a global existence theorem in a well-chosen time coordinate. In other words, a time coordinate is chosen that is geometrically defined and that, under ideal circumstances, will take all values in a certain interval $\left(t_{-}, t_{+}\right)$. The aim is then to show that, in the maximal Cauchy development of data belonging to a certain class, a time coordinate of the given type exists and exhausts the expected interval. The first result of this kind for inhomogeneous spacetimes was proved by Moncrief in [246]. This result concerned Gowdy spacetimes. These are vacuum spacetimes with a two-dimensional Abelian group of isometries acting on compact orbits. The area of the orbits defines a natural time coordinate (areal time coordinate). Moncrief showed that in the maximal Cauchy development of data given on a hypersurface of constant time, this time coordinate takes on the maximal possible range, namely $(0, \infty)$. This result was extended to more general vacuum spacetimes with two Killing vectors in [50]. Andréasson [16] extended it in another direction to the case of collisionless matter in a spacetime with Gowdy symmetry. This development was completed in [20] where general cosmological solutions of the Einstein-Vlasov system with two commuting spacelike Killing vectors were treated. Corresponding results for spacetimes with hyperbolic symmetry were obtained in [19].

In all of these cases other than Gowdy the areal time coordinate was proved to cover the 
maximal globally hyperbolic development, but the range of the coordinate was only shown to be $\left(R_{0}, \infty\right)$ for an undetermined constant $R_{0}>0$. It was not known whether $R_{0}$ was necessarily zero except in the Gowdy case. This issue was settled in [195] for the vacuum case with two commuting Killing vectors and this was extended to include Vlasov matter in [348]. It turns out that in vacuum $R_{0}=0$ apart from the exceptional case of the flat Kasner solution and an unconventional choice of the two Killing vectors. With Vlasov matter and a distribution function which does not vanish identically, $R_{0}=0$ without exception. The corresponding result in cosmological models with spherical symmetry was proved in [336] where the case of a negative cosmological constant was also included. For solutions of the Einstein-Vlasov system with hyperbolic symmetry the question is still open, although the homogeneous case was treated in [336].

Another attractive time coordinate is constant mean curvature (CMC) time. For a general discussion of this see [292]. A global existence theorem in this time for spacetimes with two Killing vectors and certain matter models (collisionless matter, wave maps) was proved in [295]. That the choice of matter model is important for this result was demonstrated by a global non-existence result for dust in [294]. As shown in [194], this leads to examples of spacetimes that are not covered by a CMC slicing. Results on global existence of CMC foliations have also been obtained for spherical and hyperbolic symmetry [289, 72].

A drawback of the results on the existence of $\mathrm{CMC}$ foliations just cited is that they require as a hypothesis the existence of one CMC Cauchy surface in the given spacetime. More recently, this restriction has been removed in certain cases by Henkel using a generalization of CMC foliations called prescribed mean curvature (PMC) foliations. A PMC foliation can be built that includes any given Cauchy surface [178] and global existence of PMC foliations can be proved in a way analogous to that previously done for CMC foliations $[177,176]$. These global foliations provide barriers that imply the existence of a CMC hypersurface. Thus, in the end it turns out that the unwanted condition in the previous theorems on CMC foliations is in fact automatically satisfied. Connections between areal, CMC, and PMC time coordinates were further explored in [19]. One important observation there is that hypersurfaces of constant areal time in spacetimes with symmetry often have mean curvature of a definite sign. Related problems for the Einstein equations coupled to fields motivated by string theory have been studied by Narita [252, 253, 254, 255].

Once global existence has been proved for a preferred time coordinate, the next step is to investigate the asymptotic behaviour of the solution as $t \rightarrow t_{ \pm}$. There are few cases in which this has been done successfully. Notable examples are Gowdy spacetimes [113, 190, 116] and solutions of the Einstein-Vlasov system with spherical and plane symmetry [272]. These last results have been extended to allow a non-zero cosmological constant in [336]. Progress in constructing spacetimes with prescribed singularities will be described in Section 6. In the future this could lead in some cases to the determination of the asymptotic behaviour of large classes of spacetimes as the singularity is approached. Detailed information has been obtained on the late-time behaviour of a class of inhomogeneous solutions of the Einstein-Vlasov system with positive cosmological constant in [337, 338] (see Section 7.6).

In the case of polarized Gowdy spacetimes a description of the late-time asymptotics was given in [116]. A proof of the validity of the asymptotic expansions can be found in [199]. The central object in the analysis of these spacetimes is a function $P$ that satisfies the equation $P_{t t}+t^{-1} P_{t}=$ $P_{\theta \theta}$. The picture that emerges is that the leading asymptotics are given by $P=A \log t+B$ for constants $A$ and $B$, this being the form taken by this function in a general Kasner model, while the next order correction consists of waves whose amplitude decays like $t^{-1 / 2}$, where $t$ is the usual Gowdy time coordinate. The entire spacetime can be reconstructed from $P$ by integration. It turns out that the generalized Kasner exponents converge to $(1,0,0)$ for inhomogeneous models. This shows that if it is stated that these models are approximated by Kasner models at late times it is necessary to be careful in what sense the approximation is supposed to hold.

General (non-polarized) Gowdy models, which are technically much more difficult to handle,

Living Reviews in Relativity

http://www. livingreviews . org/lrr-2005-6 
have been analysed in [316]. Interesting and new qualitative behaviour was found. This is one of the rare examples where a rigorous mathematical approach has discovered phenomena which had not previously been suspected on the basis of heuristic and numerical work. In the general Gowdy model the function $P$ is joined by a function $Q$ and these two functions satisfy a coupled system of nonlinear wave equations. Assuming periodic boundary conditions the solution at a fixed time $t$ defines a closed loop in the $(P, Q)$ plane. (In fact it is natural to interpret it as the hyperbolic plane.) Thus the solution as a whole can be represented by a loop which moves in the hyperbolic plane. On the basis of what happens in the polarized case it might be expected that the following would happen at late times. The diameter of the loop shrinks like $t^{-1 / 2}$ while the centre of the loop, defined in a suitable way, moves along a geodesic. In [316] Ringström shows that there are solutions which behave in the way described but there are also just as many solutions which behave in a quite different way. The shrinking of the diameter is always valid but the way the resulting small loop moves is different. There are solutions where it converges to a circle in the hyperbolic plane which is not a geodesic and it continues to move around this circle forever. A physical interpretation of this behaviour does not seem to be known.

Ringström has also obtained important new results on the structure of singularities in Gowdy spacetimes. They are discussed in Section 8.4. 


\section{Newtonian Theory and Special Relativity}

To put the global results discussed in this article into context it is helpful to compare with Newtonian theory and special relativity. Some of the theorems that have been proved in those contexts and that can offer insight into questions in general relativity will now be reviewed. It should be noted that even in these simpler contexts open questions abound.

\subsection{Hydrodynamics}

Solutions of the classical (compressible) Euler equations typically develop singularities, i.e. discontinuities of the basic fluid variables, in finite time [322]. Some of the results of [322] were recently generalized to the case of a relativistic fluid [167]. The proofs of the development of singularities are by contradiction and so do not give information about what happens when the smooth solution breaks down. One of the things that can happen is the formation of shock waves and it is known that, at least in certain cases, solutions can be extended in a physically meaningful way beyond the time of shock formation. The extended solutions only satisfy the equations in the weak sense. For the classical Euler equations there is a well-known theorem on global existence of weak solutions in one space dimension which goes back to [157]. This has been generalized to the relativistic case. Smoller and Temple treated the case of an isentropic fluid with linear equation of state [323] while Chen analysed the cases of polytropic equations of state [84] and flows with variable entropy [85]. This means that there is now an understanding of this question in the relativistic case similar to that available in the classical case.

In space dimensions higher than one there are no general global existence theorems. For a long time there were also no uniqueness theorems for weak solutions even in one dimension. It should be emphasized that weak solutions can easily be shown to be non-unique unless they are required to satisfy additional restrictions such as entropy conditions. A reasonable aim is to find a class of weak solutions in which both existence and uniqueness hold. In the one-dimensional case this has recently been achieved by Bressan and collaborators (see [68, 70, 69] and references therein).

It would be desirable to know more about which quantities must blow up when a singularity forms in higher dimensions. A partial answer was obtained for classical hydrodynamics by Chemin [83]. The possibility of generalizing this to relativistic and self-gravitating fluids was studied by Brauer [66]. There is one situation in which a smooth solution of the classical Euler equations is known to exist for all time. This is when the initial data are small and the fluid initially is flowing uniformly outwards. A theorem of this type has been proved by Grassin [161]. There is also a global existence result due to Guo [164] for an irrotational charged fluid in Newtonian physics, where the repulsive effect of the charge can suppress the formation of singularities.

A question of great practical interest for physics is that of the stability of equilibrium stellar models. The linear stability of a large class of static spherically symmetric solutions of the Einstein-Euler equations within the class of spherically symmetric perturbations has been proved by Makino [240] (cf. also [228] for the Newtonian problem). A nonlinear stability result for solutions of the Euler-Poisson system was proved in [276] under the assumption of global existence. The spectral properties of the linearized operator for general (i.e. non-spherically symmetric) perturbations in the Newtonian problem have been studied by Beyer [54]. This could perhaps provide a basis for a stability analysis, but this has not been done.

\subsection{Kinetic theory}

Collisionless matter is known to admit a global singularity-free evolution in many cases. For self-gravitating collisionless matter, which is described by the Vlasov-Poisson system, there is a general global existence theorem $[266,236]$. There is also a version of this which applies to

Living Reviews in Relativity

http://www . livingreviews . org//rr-2005-6 
Newtonian cosmology [280]. A more difficult case is that of the Vlasov-Maxwell system, which describes charged collisionless matter. Global existence is not known for general data in three space dimensions, but has been shown in two space dimensions $[154,155]$ and in three dimensions with one symmetry [153] or with almost spherically symmetric data [270].

A model system which has attracted some interest (see [18]) is the Nordström-Vlasov system where the Vlasov equation is coupled to a scalar field as in Nordström's theory of gravitation. This is not a physically correct model but may be useful for obtaining mathematical insights. A similar procedure was used to look for numerical insights in [321]. At the moment the state of knowledge concerning this system can be summed up by saying that it is roughly equal to that available for the Vlasov-Maxwell system.

The nonlinear stability of static solutions of the Vlasov-Poisson system describing Newtonian self-gravitating collisionless matter has been investigated using the energy-Casimir method. For information on this see [165] and its references. The energy-Casimir method has been applied to the Einstein equations in [350].

For the classical Boltzmann equation, global existence and uniqueness of smooth solutions has been proved for homogeneous initial data and for data that are small or close to equilibrium. For general data with finite energy and entropy, global existence of weak solutions (without uniqueness) was proved by DiPerna and Lions [132]. For information on these results and on the classical Boltzmann equation in general see $[79,80]$. Despite the non-uniqueness it is possible to show that all solutions tend to equilibrium at late times. This was first proved by Arkeryd [27] by nonstandard analysis and then by Lions [235] without those techniques. It should be noted that since the usual conservation laws for classical solutions are not known to hold for the DiPerna-Lions solutions, it is not possible to predict which equilibrium solution a given solution will converge to. In the meantime, analogues of several of these results for the classical Boltzmann equation have been proved in the relativistic case. Global existence of weak solutions was proved in [134]. Global existence and convergence to equilibrium for classical solutions starting close to equilibrium was proved in [156]. On the other hand, global existence of classical solutions for small initial data is not known. Convergence to equilibrium for weak solutions with general data was proved by Andréasson [15]. Until recently there was no existence and uniqueness theorem in the literature for general spatially homogeneous solutions of the relativistic Boltzmann equation. A paper claiming to prove existence and uniqueness for solutions of the Einstein-Boltzmann system which are homogeneous and isotropic [250] contains fundamental errors. These problems were corrected in [263] and a global existence theorem for the special relativistic Boltzmann equation was obtained. In [262] this was generalized to a global existence theorem for LRS Bianchi type I solutions of the Einstein-Boltzmann system.

Further information on kinetic theory and its relation to general relativity can be found in the Living Review of Andréasson [17].

\subsection{Elasticity theory}

There is an extensive literature on mathematical elasticity theory but the mathematics of selfgravitating elastic bodies seems to have been largely neglected. An existence theorem for spherically symmetric elastic bodies in general relativity was mentioned in Section 3.1. More recently, Beig and Schmidt [42] proved an existence theorem for static elastic bodies subject to Newtonian gravity, which need not be spherically symmetric. This was extended to rotating bodies and special relativity in [44]. 


\section{Global Existence for Small Data}

An alternative to symmetry assumptions is provided by "small data" results, where solutions are studied that develop from data close to those for known solutions. This leads to some simplification in comparison to the general problem, but with present techniques it is still very hard to obtain results of this kind.

\subsection{Stability of de Sitter space}

In [141], Friedrich proved a result on the stability of de Sitter space. He gives data at infinity but the same type of argument can be applied starting from a Cauchy surface in spacetime to give an analogous result. This concerns the Einstein vacuum equations with positive cosmological constant and is as follows. Consider initial data induced by de Sitter space on a regular Cauchy hypersurface. Then all initial data (vacuum with positive cosmological constant) near enough to these data in a suitable (Sobolev) topology have maximal Cauchy developments that are geodesically complete. The result gives much more detail on the asymptotic behaviour than just this and may be thought of as proving a form of the cosmic no hair conjecture in the vacuum case. (This conjecture says roughly that the de Sitter solution is an attractor for expanding cosmological models with positive cosmological constant.) This result is proved using conformal techniques and, in particular, the regular conformal field equations developed by Friedrich. An alternative proof of this result which extends to all higher even dimensions was given in [6]. For some comments on the case of odd dimensions see [304].

There are results obtained using the regular conformal field equations for negative or vanishing cosmological constant [143, 146], but a detailed discussion of their nature would be out of place here (cf. however Section 9.1).

\subsection{Stability of Minkowski space}

Another result on global existence for small data is that of Christodoulou and Klainerman on the stability of Minkowski space [108]. The formulation of the result is close to that given in Section 5.1, but now de Sitter space is replaced by Minkowski space. Suppose then that initial data for the vacuum Einstein equations are prescribed that are asymptotically flat and sufficiently close to those induced by Minkowski space on a hyperplane. Then Christodoulou and Klainerman prove that the maximal Cauchy development of these data is geodesically complete. They also provide a wealth of detail on the asymptotic behaviour of the solutions. The proof is very long and technical. The central tool is the Bel-Robinson tensor, which plays an analogous role for the gravitational field to that played by the energy-momentum tensor for matter fields. Apart from the book of Christodoulou and Klainerman itself, some introductory material on geometric and analytic aspects of the proof can be found in [65, 107], respectively. The result for the vacuum Einstein equations was generalized to the case of the Einstein-Maxwell system by Zipser [354].

In the original version of the theorem, initial data had to be prescribed on all of $\mathbf{R}^{3}$. A generalization described in [211] concerns the case where data need only be prescribed on the complement of a compact set in $\mathbf{R}^{3}$. This means that statements can be obtained for any asymptotically flat spacetime where the initial matter distribution has compact support, provided attention is confined to a suitable neighbourhood of infinity. The proof of the new version uses a double null foliation instead of the foliation by spacelike hypersurfaces previously used and leads to certain conceptual simplifications. A detailed treatment of this material can be found in the book of Klainerman and Nicolò [212].

An aspect of all this work which seemed less than optimal was the following. Well-known heuristic analyses by relativists produced a detailed picture of the fall-off of radiation fields in

Living Reviews in Relativity

http://www . livingreviews . org//rr-2005-6 
asymptotically flat solutions of the Einstein equations, known as peeling. It says that certain components of the Weyl tensor decay at certain rates. The analysis of Christodoulou and Klainerman reproduced some of these fall-off rates but not all. More light was shed on this discrepancy by Klainerman and Nicolò [213] who showed that if the fall-off conditions on the initial data assumed in [108] are strengthened somewhat then peeling can be proved.

A much shorter proof of the stability of Minkowski space has been given by Lindblad and Rodnianski [233]. It uses harmonic coordinates and so is closer to the original local existence proof of Choquet-Bruhat. The fact that this approach was not used earlier is related to the fact that the null condition, an important structural condition for nonlinear wave equations which implies global existence for small data, is not satisfied by the Einstein equations written in harmonic coordinates. Lindblad and Rodnianski formulated a generalization called the weak null condition [232]. This is only one element which goes into the global existence proof but it does play an important role. The result of Lindblad and Rodnianski does not give as much detail about the asymptotic structure as the approach of Christodoulou and Klainerman. On the other hand it seems that the proof generalizes without difficulty to the case of the Einstein equations coupled to a massless scalar field.

\subsection{Stability of the (compactified) Milne model}

The interior of the light cone in Minkowski space foliated by the spacelike hypersurfaces of constant Lorentzian distance from the origin can be thought of as a vacuum cosmological model, sometimes known as the Milne model. By means of a suitable discrete subgroup of the Lorentz group it can be compactified to give a spatially compact cosmological model. With a slight abuse of terminology the latter spacetime will also be referred to here as the Milne model. The stability of the latter model has been proved by Andersson and Moncrief (see [8, 11]). The result is that, given data for the Milne model on a manifold obtained by compactifying a hyperboloid in Minkowski space, the maximal Cauchy developments of nearby data are geodesically complete in the future. Moreover, the Milne model is asymptotically stable in the sense that any other solution in this class converges towards the Milne model in terms of suitable dimensionless variables.

The techniques used by Andersson and Moncrief are similar to those used by Christodoulou and Klainerman. In particular, the Bel-Robinson tensor is crucial. However, their situation is much simpler than that of Christodoulou and Klainerman, so that the complexity of the proof is not so great. This has to do with the fact that the fall-off of the fields towards infinity in the Minkowksi case is different in different directions, while it is uniform in the Milne case. Thus it is enough in the latter case to always contract the Bel-Robinson tensor with the same timelike vector when deriving energy estimates. The fact that the proof is simpler opens up a real possibility of generalizations, for instance by adding different matter models.

\subsection{Stability of the Bianchi type III form of flat spacetime}

Another vacuum cosmological model whose nonlinear stability has been investigated is the Bianchi III form of flat spacetime. To obtain this model, first do the construction described in Section 5.3 with the difference that the starting solution is three-dimensional Minkowski space. Then, take the metric product of the resulting three-dimensional Lorentz manifold with a circle. This defines a flat spacetime that has one Killing vector, which is the generator of rotations of the circle. It has been shown by Choquet-Bruhat and Moncrief [94] that this solution is stable under small vacuum perturbations preserving the one-dimensional symmetry. More precisely, they proved the result only for the polarized case. This restriction was lifted in [88]. As in the case of the Milne model, a natural task is to generalize this result to spacetimes with suitable matter content. It has been generalized to the Einstein-Maxwell-Higgs system in [89]. The reasons it is necessary to restrict 
to symmetric perturbations in this analysis, in contrast to what happens with the Milne model, are discussed in detail in [94].

One of the main techniques used is a method of modified energy estimates that is likely to be of more general applicability. The Bel-Robinson tensor plays no role. The other main technique is based on the fact that the problem under study is equivalent to the study of the $2+1$-dimensional Einstein equations coupled to a wave map (a scalar field in the polarized case). This helps to explain why the use of the Dirichlet energy could be imported into this problem from the work of [12] on $2+1$ vacuum gravity.

Living Reviews in Relativity

http: //www . livingreviews . org/lrr-2005-6 


\section{Prescribed Asymptotics}

If it is too hard to get information on the qualitative nature of solutions by evolving from a regular initial hypersurface toward a certain limiting regime (such as a possible singularity or phase of unlimited expansion), an alternative approach is to construct spacetimes with given asymptotics. Recently, the latter method has made significant progress and the new results are presented in this section.

\subsection{Isotropic singularities}

The existence and uniqueness results discussed in this section are motivated by Penrose's Weyl curvature hypothesis. Penrose suggests that the initial singularity in a cosmological model should be such that the Weyl tensor tends to zero or at least remains bounded. There is some difficulty in capturing this by a geometric condition, and it was suggested in [160] that a clearly formulated geometric condition (which, on an intuitive level, is closely related to the original condition) is that the conformal structure should remain regular at the singularity. Singularities of this type are known as conformal or isotropic singularities.

Consider now the Einstein equations coupled to a perfect fluid with the radiation equation of state $p=\rho / 3$. Then, it has been shown [257, 258, 118] that solutions with an isotropic singularity are determined uniquely by certain free data given at the singularity. The data that can be given are, roughly speaking, half as much as in the case of a regular Cauchy hypersurface. The method of proof is to derive an existence and uniqueness theorem for a suitable class of singular hyperbolic equations. In [24] this was extended to the equation of state $p=(\gamma-1) \rho$ for any $\gamma$ satisfying $1<\gamma \leq 2$

What happens to this theory when the fluid is replaced by a different matter model? The study of the case of a collisionless gas of massless particles was initiated in [25]. The equations were put into a form similar to that which was so useful in the fluid case and therefore likely to be conducive to proving existence theorems. Then theorems of this kind were proved in the homogeneous special case. These were extended to the general (i.e. inhomogeneous) case in [23]. The picture obtained for collisionless matter is very different from that for a perfect fluid. Much more data can be given freely at the singularity in the collisionless case.

These results mean that the problem of isotropic singularities has largely been solved. There do, however, remain a couple of open questions. What happens if the massless particles are replaced by massive ones? What happens if the matter is described by the Boltzmann equation with nontrivial collision term? Does the result in that case look more like the Vlasov case or more like the Euler case? A formal power series analysis of this last question was given in [342]. It was found that the asymptotic behaviour depends very much on the growth of the collision kernel for large values of the momenta.

\subsection{Fuchsian equations}

The singular equations that arise in the study of isotropic singularities are closely related to what Kichenassamy [202] calls Fuchsian equations. He has developed a rather general theory of these equations (see [202, 201, 200], and also the earlier papers [31, 203, 204]). In [205] this was applied to analytic Gowdy spacetimes on $T^{3}$ to construct a family of vacuum spacetimes depending on the maximum number of free functions (for the given symmetry class) whose singularities can be described in detail. The symmetry assumed in that paper requires the two-surfaces orthogonal to the group orbits to be surface-forming (vanishing twist constants). In [188] a corresponding result was obtained for the class of vacuum spacetimes with polarized $U(1) \times U(1)$ symmetry and nonvanishing twist. The analyticity requirement on the free functions in the case of Gowdy spacetimes 
on $T^{3}$ was reduced to smoothness in [300]. There are also Gowdy spacetimes on $S^{3}$ and $S^{2} \times S^{1}$, which have been less studied than those on $T^{3}$. The Killing vectors have zeros, defining axes, and these lead to technical difficulties. In [325] Fuchsian techniques were applied to Gowdy spacetimes on $S^{3}$ and $S^{2} \times S^{1}$. The maximum number of free functions was not obtained due to difficulties on the axes.

In [192] solutions of the vacuum Einstein equations with $U(1)$ symmetry and controlled singularity structure were constructed. They are required to satisfy some extra conditions, being polarized or half-polarized. Without these conditions oscillations are expected. The result was generalized to a larger class of topologies in [92].

Anguige [21] has obtained results on solutions with perfect fluid that are general under the condition of plane symmetry, which is stronger than Gowdy symmetry. He also extended this to polarized Gowdy symmetry in [22].

Work related to these Fuchsian methods was done earlier in a somewhat simpler context by Moncrief [247], who showed the existence of a large class of analytic vacuum spacetimes with Cauchy horizons.

As a result of the BKL picture, it cannot be expected that the singularities in general solutions of the Einstein equations in vacuum or with a non-stiff fluid can be handled using Fuchsian techniques (cf. Section 8.1). However, things look better in the presence of a massless scalar field or a stiff fluid. For these types of matter it has been possible [13] to prove a theorem analogous to that of [205] without requiring symmetry assumptions. The same conclusion can be obtained for a scalar field with mass or with a potential of moderate growth [299].

The results included in this review concern the Einstein equations in four spacetime dimensions. Of course, many of the questions discussed have analogues in other dimensions and these may be of interest for string theory and related topics. In [130] Fuchsian techniques were applied to the Einstein equations coupled to a variety of field theoretic matter models in arbitrary dimensions. One of the highlights is the result that it is possible to apply Fuchsian techniques without requiring symmetry assumptions to the vacuum Einstein equations in spacetime dimension at least eleven. Many new results are also obtained in four dimensions. For instance, the Einstein-Maxwell-dilaton and Einstein-Yang-Mills equations are treated. The general nature of the results is that, provided certain inequalities are satisfied by coupling constants, solutions with prescribed singularities can be constructed that depend on the same number of free functions as the general solution of the given Einstein-matter system. Other results on models coming from string theory have been obtained by Fuchsian methods in [256, 254, 255].

\subsection{Asymptotics for a phase of accelerated expansion}

Fuchsian techniques cannot only be used to construct singular spacetimes; they can also be used to construct spacetimes which are future geodesically complete and which exhibit accelerated expansion at late times. A solution of the Einstein equations with a foliation of spacelike hypersurfaces whose mean curvature tr $k$ is negative can be thought of as an expanding cosmological model. Supposing, for simplicity, that the hypersurfaces are compact their volume $V(t)$ satisfies $d V / d t=-(\operatorname{tr} k) V$. Associated to the volume $V$ is a length scale $l=V^{1 / 3}$, (This formula applies to the case of three space dimensions. In $n$ dimensions it should be $l=V^{1 / n}$.) Expansion corresponds to $i>0$ which is equivalent to $\operatorname{tr} k<0$. The defining condition for accelerated expansion is $\ddot{l}>0$. This is equivalent to $-d / d t(\operatorname{tr} k)+\frac{1}{3}(\operatorname{tr} k)^{2}>0$. If the leaves of the foliation are not compact this can be taken as the definition of accelerated expansion.

In [304] Fuchsian techniques were used to construct solutions of the Einstein vacuum equations with positive cosmological constant in any dimension which have accelerated expansion at late times and are not assumed to have any symmetry. Detailed asymptotic expansions are obtained for the late-time behaviour of these solutions. In the case of three spacetime dimensions these

Living Reviews in Relativity

http: //www . livingreviews . org/lrr-2005-6 
expansions were first written down by Starobinsky [326]. These spacetimes are closely related to those discussed in Section 5.1. In even spacetime dimensions they have asymptotic expansions in powers of $e^{-H t}$ where $H=\sqrt{\Lambda / 3}$, but in odd dimensions there are in general terms containing a positive power of $t$ multiplied by a power of $e^{-H t}$. 


\section{$7 \quad$ Asymptotics of Expanding Cosmological Models}

The aim of this section is to present a picture of the dynamics of forever-expanding cosmological models, by which we mean spacetimes that are maximal globally hyperbolic developments and which can be covered by a foliation by Cauchy surfaces whose mean curvature tr $k$ is strictly negative. In contrast to the approach to the big bang considered in Section 8, the spatial topology can be expected to play an important role in the present considerations. Intuitively, it may well happen that gravitational waves have time to propagate all the way around the universe. It will be assumed, as the simplest case, that the spacetimes considered admit a compact Cauchy surface. Then the hypersurfaces of negative mean curvature introduced above have finite volume and this volume is a strictly increasing function of time.

Models with accelerated expansion are now an important subject in cosmology. They have been important for a long time in connection with the early universe, where inflation plays a key role. More recently they have acquired a new significance in view of accumulating observational evidence that the expansion of the universe is accelerating at the present epoch. For these reasons it is particularly interesting to consider solutions of the Einstein equations which are appropriate for modelling cosmic acceleration. The last four Sections 7.3 to 7.6 are devoted to various aspects of this topic. An introductory account which describes some of the physical background can be found in [305].

\subsection{Lessons from homogeneous solutions}

Which features should we focus on when thinking about the dynamics of forever expanding cosmological models? Consider for a moment the Kasner solution

$$
-d t^{2}+t^{2 p_{1}} d x^{2}+t^{2 p_{2}} d y^{2}+t^{2 p_{3}} d z^{2}
$$

where $p_{1}+p_{2}+p_{3}=1$ and $p_{1}^{2}+p_{2}^{2}+p_{3}^{2}=1$. These are the first and second Kasner relations. They imply that not all $p_{i}$ can be strictly positive. Taking the coordinates $x, y$, and $z$ to be periodic gives a vacuum cosmological model whose spatial topology is that of a three-torus. The volume of the hypersurfaces $t=$ const. grows monotonically. However, the geometry does not expand in all directions, since not all $p_{i}$ are positive. This can be reformulated in a way which is more helpful when generalizing to inhomogeneous models. In fact the quantities $p_{i}$ are the eigenvalues of the second fundamental form divided by the mean curvature. The statement then is that the second fundamental form is not negative definite. Looking at other homogeneous models indicates that this behaviour of the Kasner solution is not typical of what happens more generally. On the contrary, it seems reasonable to conjecture that in general the second fundamental form eventually becomes negative definite, at least in the presence of matter.

Some examples will now be presented. The following discussion makes use of the Bianchi classification of homogenous cosmological models (see, e.g., [344]). If we take the Kasner solution and add a perfect fluid with equation of state $p=(\gamma-1) \rho, 1 \leq \gamma<2$, maintaining the symmetry (Bianchi type I), then the eigenvalues $\lambda_{i}$ of the second fundamental satisfy $\lambda_{i} / \operatorname{tr} k \rightarrow 1 / 3$ in the limit of infinite expansion. The solution isotropizes. More generally this does not happen. If we look at models of Bianchi type II with non-tilted perfect fluid, i.e. where the fluid velocity is orthogonal to the homogeneous hypersurfaces, then the quantities $p_{i}=\lambda_{i} / \operatorname{tr} k$ converge to limits that are positive but differ from 1/3 (see [344], Page 138.) There is partial but not complete isotropization. The quantities $p_{i}$ just introduced are called generalized Kasner exponents, since in the case of the Kasner solution they reduce to the $p_{i}$ in the metric form (3). This kind of partial isotropization, ensuring the definiteness of the second fundamental form at late times, seems to be typical.

Living Reviews in Relativity

http: //www . livingreviews . org/lrr-2005-6 
Intuitively, a sufficiently general vacuum spacetime should resemble gravitational waves propagating on some metric describing the large-scale geometry. This could even apply to spatially homogeneous solutions, provided they are sufficiently general. Hence, in that case also there should be partial isotropization. This expectation is confirmed in the case of vacuum spacetimes of Bianchi type VIII [313]. In that case the generalized Kasner exponents converge to non-negative limits different from $1 / 3$. For a vacuum model this can only happen if the quantity $\hat{R}=R /(\operatorname{tr} k)^{2}$, where $R$ is the spatial scalar curvature, does not tend to zero in the limit of large time. Detailed asymptotics for these spacetimes has been obtained in [314].

The Bianchi models of type VIII are the most general indefinitely expanding models of class A. Note, however, that models of class $\mathrm{VI}_{h}$ for all $h$ together are just as general. The latter models with perfect fluid and equation of state $p=(\gamma-1) \rho$ sometimes tend to the Collins model for an open set of values of $h$ for each fixed $\gamma$ (cf. [344], Page 160). These models do not in general exhibit partial isotropization. It is interesting to ask whether this is connected to the issue of spatial boundary conditions. General models of class B cannot be spatially compactified in such a way as to be locally spatially homogeneous while models of Bianchi type VIII can. See also the discussion in [33].

Another issue is what assumptions on matter are required in order that it have the effect of (partial) isotropization. Consider the case of Bianchi I. The case of a perfect fluid has already been mentioned. Collisionless matter described by kinetic theory also leads to isotropization (at least under the assumption of reflection symmetry), as do fluids with almost any physically reasonable equation of state [293]. There is, however, one exception. This is the stiff fluid, which has a linear equation of state with $\gamma=2$. In that case the generalized Kasner exponents are time-independent, and may take on negative values. In a model with two non-interacting fluids with linear equation of state the one with the smaller value of $\gamma$ dominates the dynamics at late times [120], and so the isotropization is restored. Consider now the case of a magnetic field and a perfect fluid with linear equation of state. A variety of cases of Bianchi types I, II, and $\mathrm{VI}_{0}$ have been studied in [221, 222, 223], with a mixture of rigorous results and conjectures being obtained. The general picture seems to be that, apart from very special cases, there is at least partial isotropization. The asymptotic behaviour varies with the parameter $\gamma$ in the equation of state and with the Bianchi type (only the case $\gamma \geq 1$ will be considered here). At one extreme, Bianchi type I models with $\gamma \leq 4 / 3$ isotropize. At the other extreme, the long time behaviour resembles that of a magnetovacuum model. This occurs for $\gamma>5 / 3$ in type I, for $\gamma>10 / 7$ in type II and for all $\gamma>1$ in type $\mathrm{VI}_{0}$. In all these cases there is partial isotropization.

Under what circumstances can a spatially homogeneous spacetime have the property that the generalized Kasner exponents are independent of time? The strong energy condition says that $R_{\alpha \beta} n^{\alpha} n^{\beta} \geq 0$ for any causal vector $n^{\alpha}$. It follows from the Hamiltonian constraint and the evolution equation for tr $k$ that if the generalized Kasner exponents are constant in time in a spacetime of Bianchi type I, then the normal vector $n^{\alpha}$ to the homogeneous hypersurfaces gives equality in the inequality of the strong energy condition. Hence the matter model is in a sense on the verge of violating the strong energy condition and this is a major restriction on the matter model.

A further question that can be posed concerning the dynamics of expanding cosmological models is whether $\hat{\rho}=\rho /(\operatorname{tr} k)^{2}$ tends to zero. This is of cosmological interest since $\hat{\rho}$ is (up to a constant factor) the density parameter $\Omega$ used in the cosmology literature. Note that it is not hard to show that $\operatorname{tr} k$ and $\rho$ each tend to zero in the limit for any model with $\Lambda=0$ which exists globally in the future and where the matter satisfies the dominant and strong energy conditions. First, it can be seen from the evolution equation for $\operatorname{tr} k$ that this quantity is monotone increasing and tends to zero as $t \rightarrow \infty$. Then it follows from the Hamiltonian constraint that $\rho$ tends to zero.

A reasonable condition to be demanded of an expanding cosmological model is that it be future geodesically complete. This has been proved for many homogeneous models in [290]. 


\subsection{Inhomogeneous solutions with $\Lambda=0$}

For inhomogeneous models with vanishing cosmological constant there is little information available about what happens in general. Fischer and Moncrief [139] have made an interesting proposal that attempts to establish connections between the evolution of a suitably conformally rescaled version of the spatial metric in an expanding cosmological model and themes in Riemannian geometry such as the Thurston geometrization conjecture [341], degeneration of families of metrics with bounded curvature [2], and the Ricci flow [169]. For further related work see [5]. A key element of this picture is the theorem on the stability of the Milne model discussed in Section 5.3. More generally, the rescaled metric is supposed to converge to a hyperbolic metric (metric of constant negative curvature) on a region that is large in the sense that the volume of its complement tends to zero. If the topology of the Cauchy surface is such that it is consistent with a metric of some Bianchi type, then the hyperbolic region will be missing and the volume of the entire rescaled metric will tend to zero. In this situation it might be expected that the metric converges to a (locally) homogeneous metric in some sense. Evidently the study of the nonlinear stability of Bianchi models is very relevant to developing this picture further.

Independently of the Fischer-Moncrief picture the study of small (but finite) perturbations of Bianchi models is an avenue for making progress in understanding expanding cosmological models. There is a large literature on linear perturbations of cosmological models and it would be desirable to determine what insights the results of this work might suggest for the full nonlinear dynamics. There has recently been important progress in understanding linear vacuum perturbations of various Bianchi models due to Tanimoto [330, 331, 332]. Just as it is interesting to know under what circumstances homogeneous cosmological models become isotropic in the course of expansion, it is interesting to know when more general models become homogeneous. This does happen in the case of small perturbations of the Milne model. On the other hand, there is an apparent obstruction in other cases. This is the Jeans instability [237,64]. A linear analysis indicates that under certain circumstances (e.g., perturbations of a flat Friedmann model) inhomogeneities grow with time. As yet there are no results on this available for the fully nonlinear case. A comparison that should be useful is that with Landau damping in plasma physics, where rigorous results are available [166].

The most popular matter model for spatially homogeneous cosmological models is the perfect fluid. Generalizing this to inhomogeneous models is problematic since formation of shocks or (in the case of dust) shell-crossing must be expected to occur. These signal an end to the interval of evolution of the cosmological model, which can be treated mathematically with known techniques. Initial steps have been taken to handle shocks in solutions of the Einstein-Euler equations, based on the techniques of classical hydrodynamics. The global existence (but not uniqueness) of plane symmetric weak solutions of a type which can accomodate shocks was proved in [32], while criteria proving the occurrence of shocks in plane symmetry were established in unpublished work of F. Ståhl and the author.

There are not too many results on future geodesic completeness for inhomogeneous cosmological models. A general criterion for geodesic completeness is given in [90]. It does not apply to cases like the Kasner solution but is well-suited to the case where the second fundamental form is eventually negative definite. It is part of the conclusions of [316] that Gowdy spacetimes on a torus are future geodesically complete. Information on the asymptotics is also available in the case of small but finite perturbations of the Milne model and the Bianchi type III form of flat spacetime, as discussed in Sections 5.3 and 5.4, respectively.

For solutions of the Einstein-Vlasov system with hyperbolic symmetry it has been shown by Rein [277] that future geodesic completeness holds for a certain open set of initial data. For solutions of the Einstein equations coupled to a massless linear scalar field with plane symmetry, future geodesic completeness has been shown by Tegankong [339].

Living Reviews in Relativity

http://www . livingreviews . org//rr-2005-6 


\subsection{Homogeneous models with $\Lambda>0$}

One important aspect of the fragmentary picture of the dynamics of expanding cosmological models presented in the last two Sections 7.1 and 7.2 is that it seems to be complicated. A situation where we can hope for a simpler, more unified picture is that where there is sufficiently strong acceleration of the cosmological expansion. This has the tendency to damp out irregularities. The simplest way of achieving this is to introduce a positive cosmological constant. Recall first that when the cosmological constant vanishes and the matter satisfies the usual energy conditions, spacetimes of Bianchi type IX recollapse [229] and so never belong to the indefinitely expanding models. When $\Lambda>0$ this is no longer true. Then Bianchi IX spacetimes show complicated features, and it has been suggested in the literature that they exhibit chaotic behaviour (cf. [131]). A more recent study [175] suggests that the claimed features of the solutions indicating the presence of chaos may be artefacts of the numerical treatment of a dynamical system which is not everywhere regular. The numerical work in [175] gives a different picture, parts of which the authors confirm by mathematical proofs. As a consequence this system, while complicated, may not be so intractable as previously feared and merits further analytical and numerical investigation.

In discussing homogeneous models in the following we restrict to Bianchi types other than type IX. Then a general theorem of Wald [346] states that any model whose matter content satisfies the strong and dominant energy conditions and which expands for an infinite proper time $t$ is such that all generalized Kasner exponents tend to $1 / 3$ as $t \rightarrow \infty$. A positive cosmological constant leads to isotropization. The mean curvature tends to the constant value $-\sqrt{3 \Lambda}$ as $t \rightarrow \infty$, while the scale factors increase exponentially.

Wald's result is only dependent on energy conditions and uses no details of the matter field equations. The question remains whether solutions corresponding to initial data for the Einstein equations with positive cosmological constant, coupled to reasonable matter, exist globally in time under the sole condition that the model is originally expanding. It can be shown that this is true for various matter models using the techniques of [290, 287]. This has been worked out in detail for the case of collisionless matter by Lee [224]. For the case of a perfect fluid with linear equation of state see [303]. Once global existence is known and a specific matter model has been chosen, details of the asymptotic behaviour of the matter fields can be determined and this was done in $[224,303]$. For instance, it was shown that the solutions of the Vlasov equation behave like dust asymptotically.

\subsection{Acceleration due to nonlinear scalar fields}

The effect of a cosmological constant can be mimicked by a suitable exotic matter field that violates the strong energy condition: for example, a nonlinear scalar field with exponential potential. In the latter case, an analogue of Wald's theorem has been proved by Kitada and Maeda in [207, 208]. For a potential of the form $e^{-\sqrt{8 \pi} \lambda \phi}$ with $\lambda<\sqrt{2}$, the qualitative picture is similar to that in the case of a positive cosmological constant. The difference is that the volume grows like a power of $t$ instead of exponentially and that the asymptotic rate of decay of various quantities is not the same as in the case with positive $\Lambda$. This is called power-law inflation. A global existence theorem for homogeneous solutions of the Einstein-Vlasov system with a nonlinear scalar field and a positive potential was proved in [225]. This applies in particular to the case of an exponential potential. The detailed asymptotics of geometry and matter for an exponential potential with $\lambda<\sqrt{2}$ were worked out in [225]. Corresponding global existence results in the case of a perfect fluid with linear equation of state are given in [303]. The behaviour of homogeneous and isotropic models with general $\lambda$ has been investigated in [168].

Our knowledge of the fundamental physics is insufficient to show which potential for the scalar field is most relevant for physics. It therefore makes sense to study the dynamics for large classes 
of potentials. A useful way of organizing the possibilities uses the 'rolling' picture. In a spatially homogeneous spacetime the scalar field satisfies

$$
\ddot{\phi}-\frac{1}{3} \operatorname{tr} k \dot{\phi}=-V^{\prime}(\phi)
$$

This resembles the equation of motion of a ball rolling on the graph of the potential $V$ with variable friction given by $\operatorname{tr} k$. Of course the evolution of $\operatorname{tr} k$ is coupled back to that of $\phi$ and so this analogy does not allow immediate conclusions. Nevertheless it gives an intuitive picture of what should happen. The ball should roll down to a minimum of the potential and settle down there, possibility oscillating as it does so.

The simplest case is where the potential has a strictly positive minimum. In [303] it was proved under some technical assumptions that a direct analogue of Wald's theorem holds. The late time behaviour of the geometry closely resembles that for a cosmological constant. The value of this effective cosmological constant is $\Lambda_{\text {eff }}=8 \pi V\left(\phi_{1}\right)$, where $\phi_{1}$ is the value where $V$ has its minimum. The asymptotic behaviour of the matter fields was determined in the case of collisionless matter and perfect fluids with a linear equation of state.

Another important case is where $V$ is everywhere positive and decreasing and tends to zero as $\phi \rightarrow \infty$. The 'rolling' picture suggests that $\phi$ should tend to infinity as $t \rightarrow \infty$. Under suitable technical assumptions this is true and information can be obtained concerning the asymptotics. The exponential potential is a borderline case. An important assumption is that $\lim _{\phi \rightarrow \infty} V^{\prime} / V<\sqrt{2}$ or, more generally $\lim \sup _{\phi \rightarrow \infty} V^{\prime} / V<\sqrt{2}$. Intuitively this says that the potential falls off no faster at infinity than an exponential potential which gives rise to power-law inflation. A theorem in [306] where this assumption is made in a set-up like that in Wald's theorem shows that there is always accelerated expansion for $t$ sufficiently large. If it is further assumed that $V^{\prime} / V \rightarrow 0$ as $\phi \rightarrow \infty$ then it is possible to say a lot more. It is found that, if $\sigma_{a b}$ is the tracefree part of the second fundamental form, $R$ is the spatial scalar curvature, and $\rho$ is the energy density of matter other than the scalar field then $\sigma_{a b} \sigma^{a b} /(\operatorname{tr} k)^{2}, R /(\operatorname{tr} k)^{2}$, and $\rho /(\operatorname{tr} k)^{2}$ tend to zero as $t \rightarrow \infty$. In the limit $t \rightarrow \infty$ the solution is approximated by one which is isotropic and spatially flat and contains no matter other than the scalar field. This kind of situation is sometimes called intermediate inflation since the potential is intermediate between a constant (corresponding to a cosmological constant) and an exponential (corresponding to power-law inflation).

If $V^{\prime} / V \rightarrow 0$ as $\phi \rightarrow \infty$ and $V^{\prime \prime} / V^{\prime}$ is bounded for large $\phi$ then it is possible to get further information. This is related to the 'slow-roll approximation'. The intuitive idea is that if the slope of the graph of $V$ is not too steep the ball will roll slowly and certain quantities will change gradually. It can be proved that asymptotically the term with second order derivatives in Equation (4) can be neglected and that the late-time behaviour is described approximately by the resulting first order equation. In fact this can be further simplified to give the equation $\dot{\phi}=-V^{\prime} / \sqrt{24 \pi V}$ for $\phi$ alone. This asymptotic description is not only interesting in itself; it gives a powerful method for determining the late time asymptotics when a specific potential has been chosen. For more details see [306].

Both models with a positive cosmological constant and models with a scalar field with exponential potential are called inflationary because the rate of expansion is increasing with time. There is also another kind of inflationary behaviour that arises in the presence of a scalar field with power law potential like $\phi^{4}$ or $\phi^{2}$. In that case the inflationary property concerns the behaviour of the model at intermediate times rather than at late times. The picture is that at late times the universe resembles a dust model without cosmological constant. This is known as reheating. The dynamics have been analysed heuristically by Belinskii et al. [46]. Part of their conclusions have been proved rigorously in [302]. Calculations analogous to those leading to a proof of isotropization in the case of a positive cosmological constant or an exponential potential have been done for a power law potential in [249]. In that case, the conclusion cannot apply to late time behaviour. Instead, some estimates are obtained for the expansion rate at intermediate times.

Living Reviews in Relativity

http://www. livingreviews.org//rr-2005-6 


\subsection{Other models for cosmic acceleration}

Nonlinear minimally coupled scalar fields were originally applied to the very early universe which is why the name inflation is often attached to them. Now many of these models have been recycled to model cosmic acceleration at later epochs up to the present day. In the latter context the name quintessence is used. More generally an exotic matter field violating the strong energy condition and leading to cosmic acceleration is often referred to as dark energy. In this section some models will be considered which go beyond the cosmological constant and ordinary quintessence. There is such a proliferation of models in the literature that the list considered here is far from complete.

A simple generalization of the scalar field models is a collection of several scalar fields $\phi^{i}$ [119]. These have kinetic energy $\sum_{i}\left(\dot{\phi}^{i}\right)^{2}$ and potential energy given by a function $V$ of all the $\phi^{i}$. If the $\phi^{i}$ are thought of as defining a mapping with values in $\mathbf{R}^{n}$ endowed with the Euclidean metric then it is easy to see a further generalization. Simply replace $\mathbf{R}^{n}$ by a Riemannian manifold $(N, h)$ and use the metric $h$ to define a kinetic energy as in a wave map or nonlinear $\sigma$-model. The unknown in the equation is then a mapping $\phi$ from spacetime to $N$ and the potential is a function on $N$. A more concrete description of $\phi$ can be obtained by using its components $\phi^{i}$ in a local coordinate chart on $N$. One type of model is called assisted inflation and has a potential which is the sum of exponentials of scalar fields. The name comes from the fact that even if each of these exponentials alone decays too fast to produce inflation they can assist each other so as to produce inflation in combination.

A more radical generalization is to consider a scalar field with Lagrangian $p(\phi, X)$ where $X=$ $-\frac{1}{2} \nabla^{\alpha} \phi \nabla_{\alpha} \phi$. This is known as $k$-essence [28]. In quintessence models the equation of motion of the scalar field is always hyperbolic so that the Einstein-matter equations have a well-posed initial value problem. Under the assumption that the potential is non-negative the dominant energy condition is always satisfied. These properties need not hold in $k$-essence models unless the function $p$ is restricted. In fact there is a motivation for considering models in which the dominant energy condition is violated. The value of $w=p / \rho$ in our universe can in principle be determined by observation. It is not far from -1 and if it happened to be less than -1 (which is consistent with the observations) then the dominant energy condition would be violated. It would be desirable to determine general conditions on $p$ which guarantee well-posedness and/or the dominant energy condition. In $k$-essence the equations of motion are in general quasilinear and not semilinear as they are in the case of quintessence. This may lead to the spontaneous formation of singularities in the matter field. It would be interesting to know under what conditions on $p$ this can be avoided.

Partial answers to the questions just raised can be found in $[152,151,138]$. An interesting class of models which seem to be relatively well-behaved are the tachyon models where $p(\phi, X)=$ $-V(\phi) \sqrt{1-2 X}$ for some non-negative potential $V$. Despite their name they have characteristics which lie inside the light cone. Specialising further to $V(\phi)=1$ gives a model equivalent to an exotic fluid, the Chaplygin gas. More information about the equivalence between different matter models can be found in [305].

When the dominant energy condition is violated new phenomena can occur. It is possible for an expanding cosmological model to end after finite proper time, something known as the big rip since before the final time all physical systems are ripped apart [74]. As this final time is approached the mean curvature tends to infinity, as does the energy density. This kind of behaviour can be seen explicitly for a fluid with $p=w \rho$ and $w<-1$. It is not clear that it is reasonable to consider such a fluid, but similar things could happen for other matter fields violating the dominant energy condition. It seems that there is no overview in the literature of what matter models are concerned.

To end this section we list without further comment some other exotic models which have been considered. There is the curvature-coupled scalar field (where there are some mathematical results [57]) and theories where the Einstein-Hilbert Lagrangian is replaced by some other function of the curvature. There are also models, different from Einstein gravity, which are motivated by 
loop quantum gravity [63] and brane-world theories [238] where the form of the Hamiltonian constraint is modified.

\subsection{Inhomogeneous spacetimes with accelerated expansion}

Consider what happens to Wald's proof in an inhomogeneous spacetime with positive cosmological constant. His arguments only use the Hamiltonian constraint and the evolution equation for the mean curvature. In Gauss coordinates spatial derivatives of the metric only enter these equations via the spatial scalar curvature in the Hamiltonian constraint. Hence, as noticed in [196], Wald's argument applies to the inhomogeneous case, provided we have a spacetime that exists globally in the future in Gauss coordinates and which has everywhere non-positive spatial scalar curvature. Unfortunately, it is hard to see how the latter condition can be verified starting from initial data. It is not clear whether there is a non-empty set of inhomogeneous initial data to which this argument can be applied.

In the vacuum case with positive cosmological constant, the result of Friedrich discussed in Section 5.1 proves isotropization of inhomogeneous spacetimes, i.e. that all generalized Kasner exponents corresponding to a suitable spacelike foliation tend to $1 / 3$ in the limit. To see this, consider (part of) the de Sitter metric in the form $-d t^{2}+e^{2 t}\left(d x^{2}+d y^{2}+d z^{2}\right)$. (Here, to simplify the algebra, we have chosen $\Lambda=3$.) This choice of the metric form, which is different from that discussed in [141], simplifies the algebra as much as possible. Letting $\tau=e^{-t}$ shows that the above metric can be written in the form $\tau^{-2}\left(-d \tau^{2}+d x^{2}+d y^{2}+d z^{2}\right)$. This exhibits the de Sitter metric as being conformal to a flat metric. In the construction of Friedrich the conformal class and conformal factor are perturbed. The corrections to the metric in terms of coordinate components are of relative order $\tau=e^{-t}$. Thus, the trace-free part of the second fundamental form decays exponentially, as desired.

Inflationary asymptotics has been proved in the case of inhomogeneous solutions of the EinsteinVlasov system with positive cosmological constant and three Killing vectors. This was done under the assumption of plane symmetry in [338] and for a restricted class of spherically symmetric solutions in [337]. The spacetimes were shown to be future geodesically complete and to have an asymptotic behaviour which resembles that of the de Sitter solution in leading order. Detailed information was obtained on the asymptotics of the matter fields. The results of [340] on local existence and continuation criteria for solutions of the Einstein-Vlasov-scalar field system can be thought of as a first step towards generalizing the results of [338] by replacing the cosmological constant by a scalar field.

There have been several numerical studies of inflation in inhomogeneous spacetimes. These are surveyed in Section 3 of [26]. An interesting effect which can occur in the inhomogeneous case is the formation of domain walls. Consider a potential which has two minima and suppose that the evolution at different spatial points decouples at late times. Then it may happen that in one spatial region the scalar field falls into one minimum of the potential while in another region it falls into the other minimum. In between the spatial derivatives must be relatively large in a small region forming the boundary of the two regions. This boundary is a domain wall. It would be very interesting to prove the formation of domain walls in some case.

There are heuristic results on the asymptotics of inhomogeneous solutions which are general in the sense that they have no symmetry and depend on the same number of free functions as the general solution. In [326] this kind of analysis was done for solutions of the Einstein equations in vacuum or coupled to a fluid with non-stiff linear equation of state. The mathematical interpretation of the formulae of [326] was elucidated in [304] where an analysis was done in the framework of formal power series. Compare also the results of Appendix B in [227]. In the vacuum case it was shown that there are large classes of solutions for which these series converge. A formal series analysis analogous to that of [304] was done for a scalar field with a positive minimum in [57]. It

Living Reviews in Relativity

http: //www . livingreviews . org//rr-2005-6 
was also extended to the case of curvature-coupled scalar fields. An analysis on the level of [326] has been done for a scalar field with an exponential potential in [251]. 


\section{Structure of General Singularities}

The aim of this section is to present a picture of the nature of singularities in general solutions of the Einstein equations. It is inspired by the ideas of Belinskii, Khalatnikov, and Lifshitz (BKL). To fix ideas, consider the case of a solution of the Einstein equations representing a cosmological model with a big bang singularity. A central idea of the BKL picture is that near the singularity the evolution at different spatial points decouples. This means that the global spatial topology of the model plays no role. The decoupled equations are ordinary differential equations. They coincide with the equations for spatially homogeneous cosmological models, so that the study of the latter is of particular significance.

\subsection{Lessons from homogeneous solutions}

In the BKL picture a Gaussian coordinate system $\left(t, x^{a}\right)$ is introduced such that the big bang singularity lies at $t=0$. It is not a priori clear whether this should be possible for very general spacetimes. A positive indication is given by the results of [13], where coordinates of this type are introduced in one very general class of spacetimes. Once these coordinates have been introduced, the BKL picture says that the solution of the Einstein equations should be approximated near the singularity by a family of spatially homogeneous solutions depending on the coordinates $x^{a}$ as parameters. The spatially homogeneous solutions satisfy ordinary differential equations in $t$.

Spatially homogeneous solutions can be classified into Bianchi and Kantowski-Sachs solutions. The Bianchi solutions in turn can be subdivided into types I to IX according to the Lie algebra of the isometry group of the spacetime. Two of the types, $\mathrm{VI}_{h}$ and $\mathrm{VII}_{h}$ are in fact one-parameter families of non-isomorphic Lie algebras labelled by $h$. The generality of the different symmetry types can be judged by counting the number of parameters in the initial data for each type. The result of this is that the most general types are Bianchi VIII, Bianchi IX, and Bianchi VI $-1 / 9$. The usual picture is that Bianchi VIII and Bianchi IX have more complicated dynamics than all other types and that the dynamics is similar in both these cases. This leads one to concentrate on Bianchi type IX and the mixmaster solution (see Section 3.2). Bianchi type $\mathrm{VI}_{-1 / 9}$ was apparently never mentioned in the work of BKL and has been largely ignored in the literature. Recently a detailed picture of the dynamics of these solutions has been obtained by Hewitt et al. [183] although the resulting dynamical system has not yet been analysed rigorously. Here we follow the majority and focus on Bianchi type IX.

Another aspect of the BKL picture is that most types of matter should become negligible near the singularity for suitably general solutions. In the case of perfect fluid solutions of Bianchi type IX with a linear equation of state, this has been proved by Ringström [311]. In the case of collisionless matter it remains an open issue, since rigorous results are confined to Bianchi types I, II, and III and Kantowski-Sachs, and have nothing to say about Bianchi type IX. If it is accepted that matter is usually asymptotically negligible then vacuum solutions become crucial. The vacuum solutions of Bianchi type IX (mixmaster solutions) play a central role. They exhibit complicated oscillatory behaviour, and essential aspects of this have been captured rigorously in the work of Ringström [312, 311] (compare Section 3.2).

Some matter fields can have an important effect on the dynamics near the singularity. A scalar field or stiff fluid leads to the oscillatory behaviour being replaced by monotone behaviour of the basic quantities near the singularity, and thus to a great simplification of the dynamics. An electromagnetic field can cause oscillatory behaviour that is not present in vacuum models or models with perfect fluid of the same symmetry type. For instance, models of Bianchi type I with an electromagnetic field show oscillatory, mixmaster-like behaviour [221]. However, it seems that this does not lead to anything essentially new. It is simply that the effects of spatial curvature in the more complicated Bianchi types can be replaced by electromagnetic fields in simpler Bianchi

Living Reviews in Relativity

http: //www . livingreviews . org/lrr-2005-6 
types.

A useful heuristic picture that systematizes much of what is known about the qualitative dynamical behaviour of spatially homogeneous solutions of the Einstein equations is the idea developed by Misner [245] of representing the dynamics as the motion of a particle in a time-dependent potential. In the approach to the singularity the potential develops steep walls where the particle is reflected. The mixmaster evolution consists of an infinite sequence of bounces of this kind.

\subsection{Inhomogeneous solutions}

Consider now inhomogeneous solutions of the Einstein equations where, according to the BKL picture, oscillations of mixmaster type are to be expected. This is for instance the case for general solutions of the vacuum Einstein equations. There is only one rigorous result to confirm the presence of these oscillations in an inhomogeneous spacetime of any type, and that concerns a family of spacetimes depending on only finitely many parameters [53]. They are obtained by applying a solution-generating technique to the mixmaster solution. Perhaps a reason for the dearth of results is that oscillations usually only occur in combination with the formation of local spatial structure discussed in Section 8.3. On the other hand, there is a rich variety of numerical and heuristic work supporting the BKL picture in the inhomogeneous case. For a review of the former see [49]. There are different heuristic procedures in the literature. For an approach using dimensionless variables and a dynamical systems picture see [14]. In this context it has been recognized that spatially self-similar solutions play an important role in understanding the structure of cosmological singularities. There is now a numerical calculation which shows mixmaster oscillations in vacuum solutions without any symmetry [150]. It uses a gauge condition proposed in [227]. Other interesting types of heuristics based on the Hamiltonian formulation of cosmology are the method of consistent potentials [52] and the refined billiard picture [129].

A situation where there is more hope of obtaining rigorous results is where the BKL picture suggests that there should be monotone behaviour near the singularity. This is the situation for which Fuchsian techniques can often be applied to prove the existence of large classes of spacetimes having the expected behaviour near the initial singularity (see Section 6.2). It would be desirable to have a stronger statement than these techniques have provided up to now. Ideally, it should be shown that a non-empty open set of solutions of the given class (by which is meant all solutions corresponding to an open set of initial data on a regular Cauchy surface) lead to a singularity of the given type. The only results of this type in the literature concern polarized Gowdy spacetimes [190], plane symmetric spacetimes with a massless scalar field [291], spacetimes with collisionless matter and spherical, plane or hyperbolic symmetry [272, 336], and a subset of general Gowdy spacetimes [317, 315]. The work of Christodoulou [98] on spherically symmetric solutions of the Einstein equations with a massless scalar field should also be mentioned in this context, although it concerns the singularity inside a black hole rather than singularities in cosmological models. Note that all these spacetimes have at least two Killing vectors so that the PDE problem to be solved reduces to an effective problem in one space dimension.

\subsection{Formation of localized structure}

Numerical calculations and heuristic methods such as those used by BKL lead to the conclusion that, as the singularity is approached, localized spatial structure will be formed. At any given spatial point the dynamics is approximated by that of a spatially homogeneous model near the singularity, and there will in general be bounces (cf. Section 8.1). However, there will be exceptional spatial points where the bounce fails to happen. This leads to a situation in which the spatial derivatives of the quantities describing the geometry blow up faster than these quantities themselves as the singularity is approached. In general spacetimes there will be infinitely many bounces before 
the singularity is reached, and so the points where the spatial derivatives are large will get more and more closely separated as the singularity is approached.

In Gowdy spacetimes only a finite number of bounces are to be expected and the behaviour is eventually monotone (no more bounces). There is only one essential spatial dimension due to the symmetry, and so large derivatives in general occur at isolated values of the one interesting spatial coordinate. Of course, these correspond to surfaces in space when the symmetry directions are restored. The existence of Gowdy solutions showing features of this kind has been proved in [310]. This was done by means of an explicit transformation that makes use of the symmetry.

The formation of spatial structure calls the BKL picture into question (cf. the remarks in [45]). The basic assumption underlying the BKL analysis is that spatial derivatives do not become too large near the singularity. Following the argument to its logical conclusion then indicates that spatial derivatives do become large near a dense set of points on the initial singularity. Given that the BKL picture has given so many correct insights, the hope that it may be generally applicable should not be abandoned too quickly. However, the problem represented by the formation of spatial structure shows that at the very least it is necessary to think carefully about the sense in which the BKL picture could provide a good approximation to the structure of general spacetime singularities. It should be kept in mind that the fact that certain derivatives become large does not necessarily mean that they have a large effect on the dynamics (cf. the discussion in [14]).

\subsection{Cosmic censorship in Gowdy spacetimes}

In as yet unpublished work Ringström has proved strong cosmic censorship for Gowdy spacetimes with the spatial topology of a torus, thus completing a quest which has been going on for twentyfive years. Since it was shown in [316] that these spacetimes are geodesically complete in the future, proving strong cosmic censorship comes down to showing that for generic initial data the corresponding maximal Cauchy development is inextendible towards the past. The method for doing this is to prove enough about the asymptotics near the singularity to show that the Kretschmann scalar blows up along past incomplete causal geodesics.

With a suitable genericity assumption (restriction to an open dense set of initial data) it is shown that the singularity has a structure which is similar to that found in the solutions constructed in [310]. An important technical tool is to show the existence of an 'asymptotic velocity'. This is a function constructed out of a given solution which allows the identification of the points at which localized spatial structure in the sense of Section 8.3 is formed.

Living Reviews in Relativity

http: //www . livingreviews . org/lrr-2005-6 


\section{$9 \quad$ Further Results}

This section collects miscellaneous results that do not fit into the main line of the exposition.

\subsection{Evolution of hyperboloidal data}

In Section 2.1, hyperboloidal initial data were mentioned. They can be thought of as generalizations of the data induced by Minkowski space on a hyperboloid. In the case of Minkowski space the solution admits a conformal compactification where a conformal boundary, null infinity, can be added to the spacetime. It can be shown that in the case of the maximal development of hyperboloidal data a piece of null infinity can be attached to the spacetime. For small data, i.e. data close to that of a hyperboloid in Minkowski space, this conformal boundary also has completeness properties in the future allowing an additional point $i_{+}$to be attached there (see [142] and references therein for more details). Making contact between hyperboloidal data and asymptotically flat initial data is much more difficult and there is as yet no complete picture. (An account of the results obtained up to now is given in [146].) If the relation between hyperboloidal and asymptotically flat initial data could be understood it would give a very different approach to the problem treated by Christodoulou and Klainerman (see Section 5.2). It might well also give more detailed information on the asymptotic behaviour of the solutions.

The results on the hyperboloidal initial value problem rely on the conformal field equations, a reformulation of the Einstein equations which only works in dimension four. There is an alternative method which works in all even dimensions not less than four and gives a new approach in four dimensions. This has been used in [7] to generalize some of the above results to higher even dimensions.

\subsection{The Newtonian limit}

Most textbooks on general relativity discuss the fact that Newtonian gravitational theory is the limit of general relativity as the speed of light tends to infinity. It is a non-trivial task to give a precise mathematical formulation of this statement. Ehlers systematized extensive earlier work on this problem and gave a precise definition of the Newtonian limit of general relativity that encodes those properties that are desirable on physical grounds (see [136].) Once a definition has been given, the question remains whether this definition is compatible with the Einstein equations in the sense that there are general families of solutions of the Einstein equations that have a Newtonian limit in the sense of the chosen definition. A theorem of this kind was proved in [288], where the matter content of spacetime was assumed to be a collisionless gas described by the Vlasov equation. (For another suggestion as to how this problem could be approached, see [149].) The essential mathematical problem is that of a family of equations, depending continuously on a parameter $\lambda$, which are hyperbolic for $\lambda \neq 0$ and degenerate for $\lambda=0$. Because of the singular nature of the limit it is by no means clear a priori that there are families of solutions that depend continuously on $\lambda$. That there is an abundant supply of families of this kind is the result of [288]. Asking whether there are families which are $k$ times continuously differentiable in their dependence on $\lambda$ is related to the issue of giving a mathematical justification of post-Newtonian approximations. The approach of [288] has not even been extended to the case $k=1$, and it would be desirable to do this. Note however that when $k$ is too large, serious restrictions arise [286]. The latter fact corresponds to the well-known divergent behaviour of higher order post-Newtonian approximations.

It may be useful for practical projects, for instance those based on numerical calculations, to use hybrid models in which the equations for self-gravitating Newtonian matter are modified by terms representing radiation damping. If we expand in terms of the parameter $\lambda$ as above then at some stage radiation damping terms should play a role. The hybrid models are obtained by truncating 
these expansions in a certain way. The kind of expansion that has just been mentioned can also be done, at least formally, in the case of the Maxwell equations. In that case a theorem on global existence and asymptotic behaviour for one of the hybrid models has been proved in [220]. These results have been put into context and related to the Newtonian limit of the Einstein equations in [219].

In the case of the Vlasov-Maxwell and Vlasov-Nordström systems the equivalent of the postNewtonian approximations have been justified rigorously up to certain orders [40, 39]. Calogero has proved a theorem on the Newtonian limit of the special relativistic Boltzmann equation [75].

\subsection{Newtonian cosmology}

Apart from the interest of the Newtonian limit, Newtonian gravitational theory itself may provide interesting lessons for general relativity. This is no less true for existence theorems than for other issues. In this context, it is also interesting to consider a slight generalization of Newtonian theory, the Newton-Cartan theory. This allows a nice treatment of cosmological models, which are in conflict with the (sometimes implicit) assumption in Newtonian gravitational theory that only isolated systems are considered. It is also unproblematic to introduce a cosmological constant into the Newton-Cartan theory.

Three global existence theorems have been proved in Newtonian cosmology. The first [67] is an analogue of the cosmic no hair theorem (cf. Section 5.1) and concerns models with a positive cosmological constant. It asserts that homogeneous and isotropic models are nonlinearly stable if the matter is described by dust or a polytropic fluid with pressure. Thus, it gives information about global existence and asymptotic behaviour for models arising from small (but finite) perturbations of homogeneous and isotropic data. The second and third results concern collisionless matter and the case of vanishing cosmological constant. The second [280] says that data that constitute a periodic (but not necessarily small) perturbation of a homogeneous and isotropic model that expands indefinitely give rise to solutions that exist globally in the future. The third [273] says that the homogeneous and isotropic models in Newtonian cosmology that correspond to a $k=-1$ Friedmann-Robertson-Walker model in general relativity are non-linearly stable.

\subsection{The characteristic initial value problem}

In the standard Cauchy problem, which has been the basic set-up for all the previous sections, initial data are given on a spacelike hypersurface. However, there is also another possibility, where data are given on one or more null hypersurfaces. This is the characteristic initial value problem. It has the advantage over the Cauchy problem that the constraints reduce to ordinary differential equations. One variant is to give initial data on two smooth null hypersurfaces that intersect transversely in a spacelike surface. A local existence theorem for the Einstein equations with an initial configuration of this type was proved in [284]. Another variant is to give data on a light cone. In that case local existence for the Einstein equations has not been proved, although it has been proved for a class of quasilinear hyperbolic equations that includes the reduced Einstein equations in harmonic coordinates [133]. For some new work on the global characteristic initial value problem see [73].

Another existence theorem that does not use the standard Cauchy problem, and which is closely connected to the use of null hypersurfaces, concerns the Robinson-Trautman solutions of the vacuum Einstein equations. In that case the Einstein equations reduce to a parabolic equation. Global existence for this equation has been proved by Chruściel [115].

Living Reviews in Relativity

http://www . livingreviews . org//rr-2005-6 


\subsection{The initial boundary value problem}

In most applications of evolution equations in physics (and in other sciences), initial conditions need to be supplemented by boundary conditions. This leads to the consideration of initial boundary value problems. It is not so natural to consider such problems in the case of the Einstein equations since in that case there are no physically motivated boundary conditions. (For instance, we do not know how to build a mirror for gravitational waves.) An exception is the case of a fluid boundary discussed in Section 2.6.

For the vacuum Einstein equations it is not a priori clear that it is even possible to find a well-posed initial boundary value problem. Thus, it is particularly interesting that Friedrich and Nagy [147] have been able to prove the well-posedness of certain initial boundary value problems for the vacuum Einstein equations. Since boundary conditions come up quite naturally when the Einstein equations are solved numerically, due to the need to use a finite grid, the results of [147] are potentially important for numerical relativity. The techniques developed there could also play a key role in the study of the initial value problem for fluid bodies (cf. Section 2.6).

\subsection{The geodesic hypothesis}

In elementary textbooks on general relativity we read that the Einstein equations imply that small bodies move on geodesics of the spacetime metric. It is very hard to make this into a mathematically precise statement which refers to actual solutions of the Einstein equations (and not just to some formal approximations). Recently a theorem relating to this question was proved by Stuart [329]. He considers a nonlinear wave equation which possesses soliton solutions in flat space. He studies families of solutions of the equations obtained by coupling a nonlinear wave equation of this kind to the Einstein equations. Initial data are chosen in such a way that as the parameter labelling the family tends to a limiting value the support of the data contracts to a point $p$. He shows that if the family is chosen appropriately then the solutions exist on a common time interval (although the data are becoming singular), that the geometry converges to a regular limit and that the support of the solutions converges to a timelike geodesic passing through $p$. 


\section{Acknowledgements}

I thank Håkan Andréasson, Bernd Brügmann, Mihalis Dafermos, Todd Oliynyk, John Wainwright, and Marsha Weaver for helpful suggestions. 


\section{References}

[1] Alinhac, S., and Gérard, P., Opérateurs pseudo-différentiels et Théorème de Nash-Moser, Savoirs Actuels, (EDP Sciences, Les Ulis, France, 1991). 2.4

[2] Anderson, M.T., "Scalar curvature and geometrization structures for 3-manifolds", in Grove, K., and Peterson, P., eds., Comparison Geometry, vol. 30 of Mathematical Sciences Research Institute Publications, 49-82, (Cambridge University Press, Cambridge, U.K.; New York, U.S.A., 1997). 7.2

[3] Anderson, M.T., "On stationary vacuum solutions to the Einstein equations", Ann. Henri Poincare, 1, 977-994, (2000). 3.1

[4] Anderson, M.T., "On the structure of solutions to the static vacuum Einstein equations", Ann. Henri Poincare, 1, 995-1042, (2000). 3.1

[5] Anderson, M.T., "Asymptotic behavior of future-complete cosmological space-times", Class. Quantum Grav., 21, S11-S28, (2004). 7.2

[6] Anderson, M.T., "Existence and stability of even dimensional asymptotically de Sitter spaces", (August, 2004). URL (cited on 17 March 2005):

http://arXiv.org/abs/gr-qc/0408072. 5.1

[7] Anderson, M.T., and Chruściel, P.T., "Asymptotically simple solutions of the vacuum Einstein equations in even dimensions", (December, 2004). URL (cited on 4 April 2005):

http://arXiv.org/abs/gr-qc/0412020. 9.1

[8] Andersson, L., "The global existence problem in general relativity", in Chruściel, P.T., and Friedrich, H., eds., The Einstein Equations and the Large Scale Behavior of Gravitational Fields: 50 Years of the Cauchy Problem in General Relativity, 71-120, (Birkhäuser, Basel, Switzerland; Boston, U.S.A., 2004). 5.3

[9] Andersson, L., Chruściel, P.T., and Friedrich, H., "On the regularity of solutions to the Yamabe equation and the existence of smooth hyperboloidal initial data for Einstein's field equations", Commun. Math. Phys., 149, 587-612, (1992). 2.1

[10] Andersson, L., and Moncrief, V., "Elliptic-hyperbolic systems and the Einstein equations", Ann. Henri Poincare, 4, 1-34, (2003). 2.3

[11] Andersson, L., and Moncrief, V., "Future Complete Vacuum Spacetimes", in Chruściel, P.T., and Friedrich, H., eds., The Einstein Equations and the Large Scale Behavior of Gravitational Fields: 50 Years of the Cauchy Problem in General Relativity, 299-330, (Birkhäuser, Basel, Switzerland; Boston, U.S.A., 2004). 5.3

[12] Andersson, L., Moncrief, V., and Tromba, A., "On the global evolution problem in 2+1 gravity", J. Geom. Phys., 23, 191-205, (1997). Related online version (cited on 4 April 2005):

http://arXiv.org/abs/gr-qc/9610013. 5.4

[13] Andersson, L., and Rendall, A.D., "Quiescent Cosmological Singularities", Commun. Math. Phys., 218, 479-511, (2001). 6.2, 8.1

[14] Andersson, L., van Elst, H., Lim, W.C., and Uggla, C., "Asymptotic silence of generic cosmological singularities", Phys. Rev. Lett., 94, 051101, (2005). 8.2, 8.3 
[15] Andréasson, H., "Regularity of the gain term and strong $L^{1}$ convergence to equilibrium for the relativistic Boltzmann equation", SIAM J. Math. Anal., 27, 1386-1405, (1996). 4.2

[16] Andréasson, H., "Global Foliations of Matter Spacetimes with Gowdy Symmetry", Commun. Math. Phys., 206, 337-365, (1999). 3.6

[17] Andréasson, H., "The Einstein-Vlasov System/Kinetic Theory", Living Rev. Relativity, 8, lrr-2005-2, (2005). URL (cited on 22 April 2005):

http://www. livingreviews.org/lrr-2005-2. 4.2

[18] Andréasson, H., Calogero, S., and Rein, G., "Global classical solutions to the spherically symmetric Nordström-Vlasov system", (August, 2003). URL (cited on 31 March 2005): http://arXiv.org/abs/gr-qc/0311027. 4.2

[19] Andréasson, H., Rein, G., and Rendall, A.D., "On the Einstein-Vlasov system with hyperbolic symmetry", Math. Proc. Camb. Phil. Soc., 134, 529-549, (2001). Related online version (cited on 28 January 2002):

http://arXiv.org/abs/gr-qc/0110089. 3.6

[20] Andréasson, H., Rendall, A.D., and Weaver, M., "Existence of CMC and constant areal time foliations in $T^{2}$ symmetric spacetimes with Vlasov matter", Commun. Part. Diff. Eq., 29, $237-262,(2004)$. $\quad 3.6$

[21] Anguige, K., "A class of plane symmetric perfect-fluid cosmologies with a Kasner-like singularity", Class. Quantum Grav., 17, 2117-2128, (2000). 6.2

[22] Anguige, K., "A class of plane symmetric perfect-fluid cosmologies with a Kasner-like singularity", Class. Quantum Grav., 17, 2117-2128, (2000). Related online version (cited on 29 January 2002):

http://arXiv.org/abs/gr-qc/0005086. 6.2

[23] Anguige, K., "Isotropic Cosmological Singularities. III. The Cauchy Problem for the Inhomogeneous Conformal Einstein-Vlasov Equations", Ann. Phys. (N.Y.), 282, 395-419, (2000). 6.1

[24] Anguige, K., and Tod, K.P., "Isotropic cosmological singularities 1: Polytropic perfect fluid spacetimes", Ann. Phys. (N.Y.), 276, 257-293, (1999). 6.1

[25] Anguige, K., and Tod, K.P., "Isotropic cosmological singularities 2: The Einstein-Vlasov system", Ann. Phys. (N.Y.), 276, 294-320, (1999). 6.1

[26] Anninos, P., "Computational Cosmology: From the Early Universe to the Large Scale Structure", Living Rev. Relativity, 4, lrr-2001-2, (2001). URL (cited on 24 January 2002):

http://www.livingreviews.org/lrr-2001-2. 7.6

[27] Arkeryd, L., "On the strong $L^{1}$ trend to equilibrium for the Boltzmann equation", Stud. Appl. Math., 87, 283-288, (1992). 4.2

[28] Armendariz-Picon, C., Mukhanov, V., and Steinhardt, P.J., "Essentials of $k$-essence", Phys. Rev. D, 53, 10351, (2001). 7.5

[29] Arnold, V.I., and Ilyashenko, Y.S., "Ordinary differential equations", in Anosov, D.V., and Arnold, V.I., eds., Dynamical Systems I: Ordinary Differential Equations and Smooth Dynamical Systems, vol. 1 of Encyclopaedia of Mathematical Sciences, 1-148, (Springer, Berlin, Germany; New York, U.S.A., 1988). 1

Living Reviews in Relativity

http: //www . livingreviews . org/lrr-2005-6 
[30] Aubin, T., Nonlinear Analysis on Manifolds. Monge-Ampère equations, vol. 252 of Grundlehren der mathematischen Wissenschaften, (Springer, Berlin, Germany; New York, U.S.A., 1982). 2.3

[31] Baouendi, M.S., and Goulaouic, C., "Remarks on the abstract form of nonlinear CauchyKovalevsky theorems", Commun. Part. Diff. Eq., 2, 1151-1162, (1977). 6.2

[32] Barnes, A.P., LeFloch, P.G., Schmidt, B.G., and Stewart, J.M., "The Glimm scheme for perfect fluids on plane-symmetric Gowdy spacetimes", Class. Quantum Grav., 21, 50435074, (2004). 7.2

[33] Barrow, J.D., and Kodama, H., "All universes great and small", Int. J. Mod. Phys. D, 10, 785-790, (2001). 7.1

[34] Bartnik, R., "Remarks on cosmological spacetimes and constant mean curvature hypersurfaces", Commun. Math. Phys., 117, 615-624, (1988). 2.1

[35] Bartnik, R., "Quasi-spherical metrics and prescribed scalar curvature", J. Differ. Geom., 37, 31-71, (1993). 2.1

[36] Bartnik, R., and Fodor, G., "On the restricted validity of the thin sandwich conjecture", Phys. Rev. D, 48, 3596-3599, (1993). 2.1

[37] Bartnik, R., and McKinnon, J., "Particlelike Solutions of the Einstein-Yang-Mills Equations", Phys. Rev. Lett., 61, 141-143, (1988). 3.1

[38] Batt, J., Faltenbacher, W., and Horst, E., "Stationary Spherically Symmetric Models in Stellar Dynamics", Arch. Ration. Mech. Anal., 93, 159-183, (1986). 3.1

[39] Bauer, S., "Post-Newtonian approximation of the Vlasov-Nordström system", (October, 2004). URL (cited on 13 April 2005):

http://arXiv.org/abs/math-ph/0410048. 9.2

[40] Bauer, S., and Kunze, M., "The Darwin approximation of the relativistic Vlasov-Maxwell system", (January, 2004). URL (cited on 13 April 2005):

http://arXiv.org/abs/math-ph/0401012. 9.2

[41] Beale, J.T., Hou, T.Y., and Lowengrub, J.S., "Growth rates for the linearized motion of fluid interfaces away from equilibrium", Commun. Pure Appl. Math., 46, 1269-1301, (1993). 2.6

[42] Beig, R., and Schmidt, B.G., "Static, self-gravitating elastic bodies", Proc. R. Soc. London, Ser. A, 459, 109-115, (2002). Related online version (cited on 8 February 2002):

http://arXiv.org/abs/gr-qc/0202024. 4.3

[43] Beig, R., and Schmidt, B.G., "Relativistic elasticity", Class. Quantum Grav., 20, 889-904, (2003). $\quad 2.5$

[44] Beig, R., and Schmidt, B.G., "Relativistic elastostatics I: bodies in rigid rotation", (November, 2004). URL (cited on 4 April 2004): http://arXiv.org/abs/gr-qc/0411145. 4.3

[45] Belinskii, V.A., "Turbulence of a gravitational field near a cosmological singularity", J. Exp. Theor. Phys. Lett., 56, 421-425, (1992). 8.3

[46] Belinskii, V.A., Grishchuk, L.P., Zeldovich, Y.B., and Khalatnikov, I.M., "Inflationary stages in cosmological models with a scalar field", Sov. Phys. JETP, 62, 195-203, (1986). 7.4 
[47] Belinskii, V.A., Khalatnikov, I.M., and Lifshitz, E.M., "Oscillatory approach to a singular point in the relativistic cosmology", Adv. Phys., 19, 525-573, (1970). 3.2

[48] Belinskii, V.A., Khalatnikov, I.M., and Lifshitz, E.M., "A general solution of the Einstein equations with a time singularity", Adv. Phys., 31, 639-667, (1982). 3.2

[49] Berger, B.K., "Numerical Approaches to Spacetime Singularities", Living Rev. Relativity, 5, lrr-2002-1, (2002). URL (cited on 28 January 2002):

http://www.livingreviews.org/lrr-2002-1. 8.2

[50] Berger, B.K., Chruściel, P.T., Isenberg, J.A., and Moncrief, V., "Global Foliations of Vacuum Spacetimes with $T^{2}$ Isometry", Ann. Phys. (N.Y.), 260, 117-148, (1997). 3.6

[51] Berger, B.K., Chruściel, P.T., and Moncrief, V., "On "Asymptotically Flat" Space-Times with $G_{2}$-Invariant Cauchy Surfaces", Ann. Phys. (N.Y.), 237, 322-354, (1995). 3.5

[52] Berger, B.K., Garfinkle, D., Isenberg, J., Moncrief, V., and Weaver, M., "The singularity in generic gravitational collapse is spacelike, local and oscillatory", Mod. Phys. Lett. A, 13, 1565-1574, (1998). 8.2

[53] Berger, B.K., and Moncrief, V., "Exact U(1) symmetric cosmologies with local Mixmaster dynamics", Phys. Rev. D, 62, 023509-1-023509-8, (2000). 8.2

[54] Beyer, H., "The spectrum of adiabatic stellar oscillations", J. Math. Phys., 36, 4792-4814, (1995). $\quad 4.1$

[55] Beyer, H., "On the stability of the Kerr metric", Commun. Math. Phys., 221, 659-676, (2001). $\quad 3.1$

[56] Bicak, J., Ledvinka, T., Schmidt, B.G., and Zofka, M., "Static fluid cylinders and their fields: global solutions", Class. Quantum Grav., 21, 1583-1608, (2004). 3.1

[57] Bieli, R., "Algebraic expansions for curvature coupled scalar field models", (April, 2005). URL (cited on 28 April 2005):

http://arXiv.org/abs/gr-qc/0504119. 7.5, 7.6

[58] Binney, J., and Tremaine, S., Galactic Dynamics, Princeton Series in Astrophysics, (Princeton University Press, Princeton, U.S.A., 1987). 3.1

[59] Bizoń, P., "Equivariant self-similar wave maps from Minkowski spacetime into 3-sphere", Commun. Math. Phys., 215, 45-56, (2000). 3.3

[60] Bizoń, P., Chmaj, T., and Tabor, Z., "Formation of singularities for equivariant $2+1$ dimensional wave maps into 2-sphere", Nonlinearity, 14, 1041-1053, (2001). 3.3

[61] Bizoń, P., and Tabor, Z., "On blowup of Yang-Mills fields", Phys. Rev. D, 64, 121701-1-4, (2001). 3.3

[62] Bizon, P., and Wasserman, A.G., "On the existence of self-similar spherically symmetric wave maps coupled to gravity", Class. Quantum Grav., 19, 3309-3322, (2002). Related online version (cited on 5 February 2002):

http://arXiv.org/abs/gr-qc/0201046. 3.3

[63] Bojowald, M., "Loop quantum cosmology: recent progress", (February, 2004). URL (cited on 21 April 2005):

http://arXiv.org/abs/gr-qc/0402053. 7.5 
[64] Börner, G., The Early Universe: Facts and Fiction, Texts and Monographs in Physics, (Springer, Berlin, Germany; New York, U.S.A., 1993), 3rd edition. 7.2

[65] Bourguignon, J.-P., "Stabilité par déformation non-linéaire de la métrique de Minkowski (d'après D. Christodoulou et S. Klainerman)", Asterisque, 201-203, 321-358, (1991). 5.2

[66] Brauer, U., Singularitäten in relativistischen Materiemodellen, Ph.D. Thesis, (Universität Potsdam, Potsdam, Germany, 1995). 4.1

[67] Brauer, U., Rendall, A.D., and Reula, O.A., "The cosmic no-hair theorem and the nonlinear stability of homogeneous Newtonian cosmological models", Class. Quantum Grav., 11, 22832296, (1994). 9.3

[68] Bressan, A., "The Unique Limit of the Glimm Scheme", Arch. Ration. Mech. Anal., 130, 205-230, (1995). 4.1

[69] Bressan, A., Hyperbolic Systems of Conservation Laws: The One-Dimensional Cauchy Problem, vol. 20 of Oxford Lecture Series in Mathematics and Its Applications, (Oxford University Press, Oxford, U.K.; New York, U.S.A., 2000). 4.1

[70] Bressan, A., and Colombo, R.M., "The Semigroup Generated by $2 \times 2$ Conservation Laws", Arch. Ration. Mech. Anal., 133, 1-75, (1995). 4.1

[71] Brodbeck, O., Heusler, M., Straumann, N., and Volkov, M., "Rotating solitons and nonrotating non-static black holes", Phys. Rev. Lett., 79, 4310-4313, (1997). 3.1

[72] Burnett, G.A., and Rendall, A.D., "Existence of maximal hypersurfaces in some spherically symmetric spacetimes", Class. Quantum Grav., 13, 111-123, (1996). 3.6

[73] Caciotta, G., and Nicolò, F., "Global characteristic problem for Einstein vacuum equations with small initial data: (I) The initial data constraints", J. Hyperbol. Differ. Equations, 2, 201-277, (2005). 9.4

[74] Caldwell, R.R., Kamionkowski, M., and Weinberg, N.N., "Phantom energy and cosmic doomsday", Phys. Rev. Lett., 91, 071301, (2003). 7.5

[75] Calogero, S., "The Newtonian limit of the relativistic Boltzmann equation", J. Math. Phys., 45, 4042-4052, (2004). 9.2

[76] Cantor, M., "A necessary and sufficient condition for York data to specify an asymptotically flat spacetime", J. Math. Phys., 20, 1741-1744, (1979). 2.1

[77] Carr, B.J., Coley, A.A., Goliath, M., Nilsson, U.S., and Uggla, C., "Critical phenomena and a new class of self-similar spherically symmetric perfect-fluid solutions", Phys. Rev. D, 61, 081502-1-5, (2000). 3.3

[78] Carr, B.J., Coley, A.A., Goliath, M., Nilsson, U.S., and Uggla, C., "The state space and physical interpretation of self-similar spherically symmetric perfect-fluid models", Class. Quantum Grav., 18, 303-324, (2001). 3.3

[79] Cercignani, C., The Boltzmann Equation and Its Applications, vol. 67 of Applied Mathematical Sciences, (Springer, Berlin, Germany; New York, U.S.A., 1994). 4.2

[80] Cercignani, C., Illner, R., and Pulvirenti, M., The Mathematical Theory of Dilute Gases, vol. 106 of Applied Mathematical Sciences, (Springer, Berlin, Germany; New York, U.S.A., 1988). 4.2 
[81] Chae, D., "Global existence of spherically symmetric solutions to the coupled Einstein and nonlinear Klein-Gordon system.", Class. Quantum Grav., 18, 4589-4605, (2001). 3.3

[82] Chae, D., "Global existence of solutions to the coupled Einstein and Maxwell-Higgs systems in the spherical symmetry", Ann. Henri Poincare, 4, 35-62, (2003). 3.3

[83] Chemin, J.-Y., "Remarques sur l'apparition de singularités dans les écoulements Euleriens compressibles", Commun. Math. Phys., 133, 323-339, (1990). 4.1

[84] Chen, J., "Conservation laws for the relativistic p-system", Commun. Part. Diff. Eq., 20, 1605-1646, (1995). 4.1

[85] Chen, J., "Conservation Laws for Relativistic Fluid Dynamics", Arch. Ration. Mech. Anal., 139, 377-398, (1997). 4.1

[86] Choptuik, M.W., "Universality and Scaling in the Gravitational Collapse of a Massless Scalar Field", Phys. Rev. Lett., 70, 9-12, (1993). 3.3

[87] Choquet-Bruhat, Y., " $C^{\infty}$ solutions of hyperbolic nonlinear equations applications to G. R. G.", Gen. Relativ. Gravit., 2, 359-362, (1971). 2.3

[88] Choquet-Bruhat, Y., "Future complete Einsteinian spacetimes with U(1) isometry group, the unpolarized case", in Chruściel, P.T., and Friedrich, H., eds., The Einstein Equations and the Large Scale Behavior of Gravitational Fields: 50 Years of the Cauchy Problem in General Relativity, 251-298, (Birkhäuser, Basel, Switzerland; Boston, U.S.A., 2004). 5.4

[89] Choquet-Bruhat, Y., "Future complete $S^{1}$ symmetric solutions of the Einstein-MaxwellHiggs system", (January, 2005). URL (cited on 1 March 2005):

http://arXiv.org/abs/gr-qc/0501052. 5.4

[90] Choquet-Bruhat, Y., and Cotsakis, S., "Global hyperbolicity and completeness", J. Geom. Phys., 43, 345-350, (2002). Related online version (cited on 20 February 2002): http://arXiv.org/abs/gr-qc/0201057. 7.2

[91] Choquet-Bruhat, Y., and Geroch, R., "Global aspects of the Cauchy problem in general relativity", Commun. Math. Phys., 14, 329-335, (1969). 2.2

[92] Choquet-Bruhat, Y., Isenberg, J.A., and Moncrief, V., "Topologically general U(1) symmetric Einstein spacetimes with AVTD behavior", (February, 2005). URL (cited on 13 April 2005):

http://arXiv.org/abs/gr-qc/0502104. 6.2

[93] Choquet-Bruhat, Y., Isenberg, J.A., and York, J.W., "Einstein constraints on asymptotically Euclidean manifolds", Phys. Rev. D, 61, 084034-1-20, (2000). 2.1

[94] Choquet-Bruhat, Y., and Moncrief, V., "Future global in time Einsteinian spacetimes with U(1) isometry group", Ann. Henri Poincare, 2, 1007-1064, (2001). 5.4

[95] Choquet-Bruhat, Y., and York, J.W., "The Cauchy problem", in Held, A., ed., General Relativity and Gravitation : One Hundred Years After the Birth of Albert Einstein, vol. 1, 99-172, (Plenum, New York, U.S.A., 1980). 2.1, 2.2

[96] Christodoulou, D., "Global existence of generalised solutions of the spherically symmetric Einstein-scalar equations in the large", Commun. Math. Phys., 106, 587-621, (1986). 3.3

Living Reviews in Relativity

http: //www. livingreviews. org/lrr-2005-6 
[97] Christodoulou, D., "The problem of a self-gravitating scalar field", Commun. Math. Phys., 105, 337-361, (1986). 3.3

[98] Christodoulou, D., "A mathematical theory of gravitational collapse", Commun. Math. Phys., 109, 613-647, (1987). 3.3, 8.2

[99] Christodoulou, D., "The structure and uniqueness of generalised solutions of the spherically symmetric Einstein-scalar equations", Commun. Math. Phys., 109, 591-611, (1987). 3.3

[100] Christodoulou, D., "The formation of black holes and singularities in spherically symmetric gravitational collapse", Commun. Pure Appl. Math., 44, 339-373, (1991). 3.3

[101] Christodoulou, D., "Bounded variation solutions of the spherically symmetric Einstein-scalar field equations", Commun. Pure Appl. Math., 46, 1131-1220, (1993). 3.3

[102] Christodoulou, D., "Examples of naked singularity formation in the gravitational collapse of a scalar field", Ann. Math., 140, 607-653, (1994). 3.3

[103] Christodoulou, D., "Self-Gravitating Fluids: A Two-Phase Model", Arch. Ration. Mech. Anal., 130, 343-400, (1995). 3.3

[104] Christodoulou, D., "Self-Gravitating Fluids: The Continuation and Termination of a Free Phase Boundary", Arch. Ration. Mech. Anal., 133, 333-398, (1996). 3.3

[105] Christodoulou, D., "Self-Gravitating Fluids: The Formation of a Free Phase Boundary in the Phase Transition from Soft to Hard", Arch. Ration. Mech. Anal., 134, 97-154, (1996). 3.3

[106] Christodoulou, D., "The instability of naked singularities in the gravitational collapse of a scalar field", Ann. Math. (2), 149, 183-217, (1999). 3.3

[107] Christodoulou, D., and Klainerman, S., "Asymptotic properties of linear field equations in Minkowski space", Commun. Pure Appl. Math., 43, 137-199, (1990). 5.2

[108] Christodoulou, D., and Klainerman, S., The global nonlinear stability of the Minkowski space, vol. 41 of Princeton Mathematical Series, (Princeton University Press, Princeton, U.S.A., 1993). $\quad 5.2$

[109] Christodoulou, D., and Lindblad, H., "On the motion of the free surface of a liquid", Commun. Pure Appl. Math., 53, 1536-1602, (2000). 2.6

[110] Christodoulou, D., and Ó Murchadha, N., "The boost problem in general relativity", Commun. Math. Phys., 80, 271-300, (1981). 2.1, 2.2

[111] Christodoulou, D., and Tahvildar-Zadeh, A.S., "On the asymptotic behaviour of spherically symmetric wave maps", Duke Math. J., 71, 31-69, (1993). 3.5

[112] Christodoulou, D., and Tahvildar-Zadeh, A.S., "On the regularity of spherically symmetric wave maps", Commun. Pure Appl. Math., 46, 1041-1091, (1993). 3.5

[113] Chruściel, P.T., "On Space-Time with $U(1) \times U(1)$ Symmetric Compact Cauchy Surfaces", Ann. Phys. (N.Y.), 202, 100-150, (1990). 3.6

[114] Chruściel, P.T., On Uniqueness in the Large of Solutions of Einstein's Equations (Strong Cosmic Censorship), vol. 27 of Proceedings of the Centre for Mathematics and its Applications, (Australian National University Press, Canberra, Australia, 1991). 1 
[115] Chruściel, P.T., "Semi-global existence and convergence of solutions of the RobinsonTrautman (2-dimensional Calabi) equation", Commun. Math. Phys., 137, 289-313, (1991). 9.4

[116] Chruściel, P.T., Isenberg, J.A., and Moncrief, V., "Strong cosmic censorship in polarised Gowdy spacetimes", Class. Quantum Grav., 7, 1671-1680, (1990). 3.6

[117] Chruściel, P.T., Isenberg, J.A., and Pollack, D., "Gluing initial data sets for general relativity", Phys. Rev. Lett., 93, 081101, (2004). 2.1

[118] Claudel, C.M., and Newman, K.P., "The Cauchy problem for quasi-linear hyperbolic evolution problems with a singularity in the time", Proc. R. Soc. London, Ser. A, 454, 1073-1107, (1998). 6.1

[119] Coley, A.A., and van den Hoogen, R.J., "The dynamics of multi-scalar field cosmological models and assisted inflation", Phys. Rev. D, 62, 023517, (2000). 7.5

[120] Coley, A.A., and Wainwright, J., "Qualitative analysis of two-fluid Bianchi cosmologies", Class. Quantum Grav., 9, 651-665, (1992). 7.1

[121] Corvino, J., "Scalar curvature deformation and a gluing construction for the Einstein constraint equations", Commun. Math. Phys., 214, 137-189, (2000). 2.1

[122] Dafermos, M., "Stability and instability of the Cauchy horizon for the spherically symmetric Einstein-Maxwell-scalar field equations", Ann. Math., 158, 875-928, (2003). 3.4

[123] Dafermos, M., "On naked singularities and the collapse of self-gravitating Higgs fields", (March, 2004). URL (cited on 4 April 2005):

http://arXiv.org/abs/gr-qc/0403033. 3.3

[124] Dafermos, M., "Spherically symmetric spacetimes with a trapped surface", (March, 2004). URL (cited on 31 March 2005): http://arXiv.org/abs/gr-qc/0403032. 3.3

[125] Dafermos, M., and Rendall, A.D., "An extension principle for the Einstein-Vlasov system in spherical symmetry", (November, 2004). URL (cited on 1 April 2005):

http://arXiv.org/abs/gr-qc/0411075. 3.3

[126] Dafermos, M., and Rodnianski, I., "A proof of Price's law for the collapse of a self-gravitating scalar field", (September, 2003). URL (cited on 31 March 2005): http://arXiv.org/abs/gr-qc/0309115. 3.4

[127] Dain, S., "Trapped surfaces as boundaries for the constraint equations", Class. Quantum Grav., 21, 555-574, (2004). 2.1

[128] Dain, S., and Nagy, G., "Initial data for fluid bodies in general relativity", Phys. Rev. D, 65, 084020-1-15, (2002). Related online version (cited on 30 January 2002):

http://arXiv.org/abs/gr-qc/0201091. 2.6

[129] Damour, T., "Cosmological singularities, Einstein billiards and Lorentzian Kac-Moody algebras", (January, 2005). URL (cited on 28 July 2005):

http://arXiv.org/abs/gr-qc/0501064. 8.2

[130] Damour, T., Henneaux, M., Rendall, A.D., and Weaver, M., "Kasner-like behaviour for subcritical Einstein-matter systems", Ann. Henri Poincare, 3, 1049-1111, (2002). URL (cited on 20 February 2002):

http://arXiv.org/abs/gr-qc/0202069. 6.2 
[131] de Oliveira, H.P., Ozorio de Almeida, A.M., Damião Soares, I., and Tonini, E.V., "Homoclinic chaos in the dynamics of a general Bianchi type-IX model", Phys. Rev. D, 65, 083511-1-9, (2002). Related online version (cited on 17 February 2002):

http://arXiv.org/abs/gr-qc/0202047. 7.3

[132] DiPerna, R.J., and Lions, P.-L., "On the Cauchy problem for Boltzmann equations: Global existence and weak stability", Ann. Math., 130, 321-366, (1989). 4.2

[133] Dossa, M., "Espaces de Sobolev non isotropes, à poids et problèmes de Cauchy quasi-linéaires sur un conoïde caractéristique", Ann. Inst. Henri Poincare A, 66, 37-107, (1997). 9.4

[134] Dudyński, M., and Ekiel-Jezewska, M.L., "Global existence proof for the relativistic Boltzmann equation", J. Stat. Phys., 66, 991-1001, (1992). 4.2

[135] Eardley, D.M., and Moncrief, V., "The global existence of Yang-Mills fields in $M^{3+1}$ ", Commun. Math. Phys., 83, 171-212, (1982). 2.3, 3.3

[136] Ehlers, J., "The Newtonian limit of general relativity", in Ferrarese, G., ed., Classical Mechanics and Relativity: Relationship and Consistency, International Conference in Memory of Carlo Cattaneo, Elba 9-13 July 1989, Monographs and Textbooks in Physical Science, (Bibliopolis, Naples, Italy, 1991). 9.2

[137] Evans, L.C., Partial Differential Equations, vol. 19 of Graduate Studies in Mathematics, (American Mathematical Society, Providence, U.S.A., 1998). 1

[138] Felder, G., Kofman, L., and Starobinsky, A.A., "Caustics in tachyon matter and other BornInfeld scalars", J. High Energy Phys., 0209, 026, (2001). 7.5

[139] Fischer, A., and Moncrief, V., "The Einstein flow, the $\sigma$-constant and the geometriztion of 3-manifolds", Class. Quantum Grav., 16, L79-L87, (1999). 7.2

[140] Fjällborg, M., "On the cylindrically symmetric Einstein-Vlasov system", (March, 2005). URL (cited on 31 March 2005): http://arXiv.org/abs/gr-qc/0503098. 3.5

[141] Friedrich, H., "Existence and structure of past asymptotically simple solutions of Einstein's field equations with positive cosmological constant", J. Geom. Phys., 3, 101-117, (1986). $5.1,7.6$

[142] Friedrich, H., "On the global existence and asymptotic behaviour of solutions to the EinsteinYang-Mills equations", J. Differ. Geom., 34, 275-345, (1991). 9.1

[143] Friedrich, H., "Einstein equations and conformal structure: Existence of anti-de Sitter-type spacetimes", J. Geom. Phys., 17, 125-184, (1995). 5.1

[144] Friedrich, H., "Hyperbolic reductions of Einstein's field equations", Class. Quantum Grav., 13, 1451-1469, (1996). 2.2

[145] Friedrich, H., "Evolution equations for gravitating ideal fluid bodies in general relativity", Phys. Rev. D, 57, 2317-2322, (1998). 2.6

[146] Friedrich, H., "Gravitational fields near spacelike and null infinity", J. Geom. Phys., 24, 83-172, (1998). $\quad 5.1,9.1$

[147] Friedrich, H., and Nagy, G., "The initial boundary value problem for Einstein's vacuum field equations", Commun. Math. Phys., 201, 619-655, (1999). 9.5 
[148] Friedrich, H., and Rendall, A.D., "The Cauchy problem for the Einstein equations", in Schmidt, B.G., ed., Einstein's Field Equations and Their Physical Implications: Selected Essays in Honour of Jürgen Ehlers, vol. 540 of Lecture Notes in Physics, (Springer, Berlin, Germany; New York, U.S.A., 2000). 2, 2.2, 2.6

[149] Fritelli, S., and Reula, O.A., "On the Newtonian limit of general relativity", Commun. Math. Phys., 166, 221-235, (1994). 9.2

[150] Garfinkle, D., "Numerical simulations of generic singuarities", Phys. Rev. Lett., 93, 124017, (2004). $\quad 8.2$

[151] Gibbons, G.W., "Phantom matter and the cosmological constant", (February, 2003). URL (cited on 21 April 2005):

http://arXiv.org/abs/gr-qc/0302199. 7.5

[152] Gibbons, G.W., "Thoughts on tachyon cosmology", Class. Quantum Grav., 20, S321-S346, (2003). $\quad 7.5$

[153] Glassey, R.T., and Schaeffer, J., "The 'two and one half dimensional' relativistic VlasovMaxwell system", Commun. Math. Phys., 185, 257-284, (1997). 4.2

[154] Glassey, R.T., and Schaeffer, J., "The Relativistic Vlasov-Maxwell System in Two Space Dimensions: Part I", Arch. Ration. Mech. Anal., 141, 331-354, (1998). 4.2

[155] Glassey, R.T., and Schaeffer, J., "The Relativistic Vlasov-Maxwell System in Two Space Dimensions: Part II", Arch. Ration. Mech. Anal., 141, 355-374, (1998). 4.2

[156] Glassey, R.T., and Strauss, W., "Asymptotic stability of the relativistic Maxwellian", Publ. Res. Inst. Math. Sci., 29, 301-347, (1993). 4.2

[157] Glimm, J., "Solutions in the large for nonlinear hyperbolic systems of equations", Commun. Pure Appl. Math., 18, 697-715, (1965). 4.1

[158] Goliath, M., Nilsson, U.S., and Uggla, C., "Spatially self-similar spherically symmetric perfect-fluid models", Class. Quantum Grav., 15, 167-186, (1998). 3.3

[159] Goliath, M., Nilsson, U.S., and Uggla, C., "Timelike self-similar spherically symmetric perfect-fluid models", Class. Quantum Grav., 15, 2841-2863, (1998). 3.3

[160] Goode, S.W., and Wainwright, J., "Isotropic singularities in cosmological models", Class. Quantum Grav., 2, 99-115, (1985). 6.1

[161] Grassin, M., "Global smooth solutions to Euler equations for a perfect gas", Indiana Univ. Math. J., 47, 1397-1432, (1998). 4.1

[162] Gundlach, C., "Critical phenomena in gravitational collapse", Adv. Theor. Math. Phys., 2, 1-49, (1998). 3.3

[163] Gundlach, C., "Critical Phenomena in Gravitational Collapse", Living Rev. Relativity, 2, lrr-1999-4, (1999). URL (cited on 22 December 1999):

http://www. livingreviews.org/lrr-1999-4. 3.3

[164] Guo, Y., "Smooth irrotational flows in the large to the Euler-Poisson system", Commun. Math. Phys., 195, 249-265, (1998). 4.1

[165] Guo, Y., and Rein, G., "Isotropic steady states in stellar dynamics", Commun. Math. Phys., 219, 607-629, (2001). 4.2

Living Reviews in Relativity

http: //www . livingreviews . org/lrr-2005-6 
[166] Guo, Y., and Strauss, W., "Nonlinear instability of double-humped equilibria", Ann. Inst. Henri Poincare C, 12, 339-352, (1995). 7.2

[167] Guo, Y., and Tahvildar-Zadeh, A.S., "Formation of singularities in relativistic fluid dynamics and in spherically symmetric plasma dynamics", in Chen, G.-Q., and DiBenedetto, E., eds., Nonlinear Partial Differential Equations, International Conference on Nonlinear Partial Differential Equations and Applications, March 21-24, 1998, Northwestern University, vol. 238 of Contemporary Mathematics, (American Mathematical Society, Providence, U.S.A., 1999). 4.1

[168] Halliwell, J.J., "Scalar fields in cosmology with an exponential potential", Phys. Lett. B, 185, 341-344, (1987). 7.4

[169] Hamilton, R., "Three manifolds of positive Ricci curvature", J. Differ. Geom., 17, 255-306, (1982). $\quad 7.2$

[170] Hartman, P., Ordinary Differential Equations, (Birkhäuser, Boston, U.S.A., 1982), 2nd edition. 1

[171] Hauser, I., and Ernst, F.J., "Proof of a generalized Geroch conjecture for the hyperbolic Ernst equation", Gen. Relativ. Gravit., 33, 195-293, (2001). 3.5

[172] Heilig, U., "On the existence of rotating stars in general relativity", Commun. Math. Phys., 166, 457-493, (1995). 3.1

[173] Heinzle, M., Rendall, A.D., and Uggla, C., "Theory of Newtonian self-gravitating stationary spherically symmetric systems", (August, 2004). URL (cited on 30 March 2005): http://arXiv.org/abs/math-ph/0408045. 3.1

[174] Heinzle, M., Röhr, N., and Uggla, C., "Spherically symmetric relativistic stellar structures", Class. Quantum Grav., 20, 4567-4586, (2003). 3.1

[175] Heinzle, M., Röhr, N., and Uggla, C., "Matter and dynamics in closed cosmologies", (June, 2004). URL (cited on 1 April 2005):

http://arXiv.org/abs/math-ph/0406072. 7.3

[176] Henkel, O., "Global prescribed mean curvature foliations in cosmological space-times. II", J. Math. Phys., 43, 2466-2485, (2001). Related online version (cited on 28 January 2002): http://arXiv.org/abs/gr-qc/0110082. 3.6

[177] Henkel, O., "Global prescribed mean curvature foliations in cosmological space-times. I", $J$. Math. Phys., 43, 2439-2465, (2002). Related online version (cited on 28 January 2002): http://arXiv.org/abs/gr-qc/0110081. 3.6

[178] Henkel, O., "Local prescribed mean curvature foliations in cosmological spacetimes", Math. Proc. Camb. Phil. Soc., 134, 551-571, (2003). Related online version (cited on 28 January 2002):

http://arXiv.org/abs/gr-qc/0108003. 3.6

[179] Hertog, T., Horowitz, G.T., and Maeda, K., "Generic cosmic censorship violation in anti de Sitter space.", Phys. Rev. Lett., 92, 131101, (2004). 3.3

[180] Hervik, S., van den Hoogen, R., and Coley, A.A., "Future asymptotic behaviour of tilted Bianchi models of type IV and VII ${ }_{h}$ ", Class. Quantum Grav., 22, 607-634, (2005). 3.2 
[181] Heusler, M., Black Hole Uniqueness Theorems, (Cambridge University Press, Cambridge, U.K.; New York, U.S.A., 1996). 3.1

[182] Heusler, M., "Stationary black holes: Uniqueness and beyond", Living Rev. Relativity, 1, lrr-1998-6, (1998). URL (cited on 12 December 1999):

http://www. livingreviews.org/lrr-1998-6. 3.1

[183] Hewitt, C., Horwood, J.T., and Wainwright, J., "Asymptotic dynamics of the exceptional Bianchi cosmologies", Class. Quantum Grav., 20, 1743-1756, (2003). 8.1

[184] Hewitt, C., and Wainwright, J., "The asymptotic regimes of tilted Bianchi II cosmologies", Gen. Relativ. Gravit., 33, 65-94, (2001). 3.2

[185] Hod, S., and Piran, T., "Mass inflation in dynamical collapse of a charged scalar field", Phys. Rev. Lett., 81, 1554-1557, (1998). 3.4

[186] Hubbard, J.H., and West, B.H., Differential Equations: A Dynamical Systems Approach. Ordinary Differential Equations, vol. 5 of Texts in Applied Mathematics, (Springer, Berlin, 1991), 3rd edition. 1

[187] Isenberg, J.A., "Constant mean curvature solutions of the Einstein constraint equations on closed manifolds", Class. Quantum Grav., 12, 2249-2274, (1995). 2.1

[188] Isenberg, J.A., and Kichenassamy, S., "Asymptotic behaviour in polarized $T^{2}$-symmetric vacuum space-times", J. Math. Phys., 40, 340-352, (1999). 6.2

[189] Isenberg, J.A., Mazzeo, R., and Pollack, D., "On the topology of vacuum spacetimes", Ann. Henri Poincare, 3, 369-383, (2003). 2.1

[190] Isenberg, J.A., and Moncrief, V., "Asymptotic Behaviour of the Gravitational Field and the Nature of Singularities in Gowdy Spacetimes", Ann. Phys. (N.Y.), 199, 84-122, (1990). 3.6, 8.2

[191] Isenberg, J.A., and Moncrief, V., "A set of nonconstant mean curvature solutions of the Einstein constraint equations on closed manifolds", Class. Quantum Grav., 13, 1819-1847, (1996). 2.1

[192] Isenberg, J.A., and Moncrief, V., "Asymptotic behavior of polarized and half-polarized U(1) symmetric vacuum spacetimes", Class. Quantum Grav., 19, 5361-5386, (2002). 6.2

[193] Isenberg, J.A., and Ó Murchadha, N., "Non CMC conformal data sets which do not produce solutions of the Einstein constraint equations", Class. Quantum Grav., 21, S233-S241, (2004). $\quad 2.1$

[194] Isenberg, J.A., and Rendall, A.D., "Cosmological spacetimes not covered by a constant mean curvature slicing", Class. Quantum Grav., 15, 3679-3688, (1998). 3.6

[195] Isenberg, J.A., and Weaver, M., "On the area of the symmetry orbits in $T^{2}$ symmetric spacetimes", Class. Quantum Grav., 20, 3783-3796, (2003). 3.6

[196] Jensen, L.G., and Stein-Schabes, J.A., "Is inflation natural?", Phys. Rev. D, 35, 1146-1150, (1987). $\quad 7.6$

[197] John, F., Partial Differential Equations, vol. 1 of Applied Mathematical Sciences, (Springer, Berlin, Germany; New York, U.S.A., 1982), 4th edition. 1

Living Reviews in Relativity

http://www. livingreviews . org/lrr-2005-6 
[198] John, F., Nonlinear Wave Equations, Formation of Singularities, vol. 2 of University Lecture Series, (American Mathematical Society, Providence, U.S.A., 1990). 1

[199] Jurke, T., "On future asymptotics of polarized Gowdy $T^{3}$-models", Class. Quantum Grav., 20, 173-192, (2003). 3.6

[200] Kichenassamy, S., "The blow-up problem for exponential nonlinearities", Commun. Part. Diff. Eq., 21, 125-162, (1996). 6.2

[201] Kichenassamy, S., "Fuchsian equations in Sobolev spaces and blow-up", J. Differ. Equations, 125, 299-327, (1996). 6.2

[202] Kichenassamy, S., Nonlinear Wave Equations, vol. 194 of Monographs and Textbooks in Pure and Applied Mathematics, (Marcel Dekker, New York, U.S.A., 1996). 1, 6.2

[203] Kichenassamy, S., and Littman, W., "Blow-up surfaces for nonlinear wave equations, I", Commun. Part. Diff. Eq., 18, 431-452, (1993). 6.2

[204] Kichenassamy, S., and Littman, W., "Blow-up surfaces for nonlinear wave equations, II", Commun. Part. Diff. Eq., 18, 1869-1899, (1993). 6.2

[205] Kichenassamy, S., and Rendall, A.D., "Analytic description of singularities in Gowdy spacetimes", Class. Quantum Grav., 15, 1339-1355, (1998). 6.2

[206] Kind, S., and Ehlers, J., "Initial boundary value problem for the spherically symmetric Einstein equations for a perfect fluid", Class. Quantum Grav., 18, 2123-2136, (1993). 2.6

[207] Kitada, Y., and Maeda, K., "Cosmic no-hair theorem in power-law inflation", Phys. Rev. D, 45, 1416-1419, (1992). 7.4

[208] Kitada, Y., and Maeda, K., "Cosmic no-hair theorem in homogeneous cosmological models. I. Bianchi models.", Class. Quantum Grav., 10, 703-734, (1993). 7.4

[209] Klainerman, S., "A commuting vector fields approach to Strichartz-type inequalities and applications to quasi-linear wave equations", Int. Math. Res. Notices, 2001(5), 221-274, (2001). $\quad 2.4$

[210] Klainerman, S., and Machedon, M., "Finite energy solutions of the Yang-Mills equations in $\mathbf{R}^{3+1}$ ", Ann. Math., 142, 39-119, (1995). 2.3, 3.3

[211] Klainerman, S., and Nicolò, F., "On local and global aspects of the Cauchy problem in general relativity", Class. Quantum Grav., 16, R73-R157, (1999). 2.2, 5.2

[212] Klainerman, S., and Nicolò, F., The evolution problem in general relativity, (Birkhäuser, Boston, U.S.A., 2003). 5.2

[213] Klainerman, S., and Nicolò, F., "Peeling properties of asymptotically flat solutions to the Einstein vacuum equations", Class. Quantum Grav., 20, 3215-3257, (2003). 5.2

[214] Klainerman, S., and Rodnianski, I., "Rough solution for the Einstein vacuum equations", (September, 2001). URL (cited on 1 March 2002): http://arXiv.org/abs/math.AP/0109173. 2.4

[215] Klainerman, S., and Rodnianski, I., "Causal geometry of Einstein-vacuum spacetimes with finite curvature flux", (September, 2003). URL (cited on 30 March 2005):

http://arXiv.org/abs/math.AP/0309459. 2.4 
[216] Klainerman, S., and Rodnianski, I., "A geometric approach to the Littlewood-Paley theory", (September, 2003). URL (cited on 30 March 2005):

http://arXiv.org/abs/math.AP/0309463. 2.4

[217] Kleihaus, B., and Kunz, J., "Static axially symmetric Einstein-Yang-Mills-dilaton solutions: I. Regular solutions", Phys. Rev. D, 57, 834-856, (1998). 3.1

[218] Krieger, J., "Stability of spherically symmetric wave maps", (March, 2005). URL (cited on 30 April 2005):

http://arXiv.org/abs/math.AP/0503048. 3.5

[219] Kunze, M., and Rendall, A.D., "Simplified models of electromagnetic and gravitational radiation damping.", Class. Quantum Grav., 18, 3573-3587, (2001). 9.2

[220] Kunze, M., and Rendall, A.D., "The Vlasov-Poisson system with radiation damping.", Ann. Henri Poincare, 2, 857-886, (2001). 9.2

[221] LeBlanc, V.G., "Asymptotic states of magnetic Bianchi I cosmologies", Class. Quantum Grav., 14, 2281-2301, (1997). 7.1, 8.1

[222] LeBlanc, V.G., "Bianchi II magnetic cosmologies", Class. Quantum Grav., 15, 1607-1626, (1998). $\quad 7.1$

[223] LeBlanc, V.G., Kerr, D., and Wainwright, J., "Asymptotic states of magnetic Bianchi $\mathrm{VI}_{0}$ cosmologies", Class. Quantum Grav., 12, 513-541, (1995). 7.1

[224] Lee, H., "Asymptotic behaviour of the Einstein-Vlasov system with a positive cosmological constant", Math. Proc. Camb. Phil. Soc., 137, 495-509, (2004). 7.3

[225] Lee, H., "The Einstein-Vlasov system with a scalar field", (April, 2004). URL (cited on 30 March 2005):

http://arXiv.org/abs/gr-qc/0404007. 7.4

[226] Lifshitz, E.M., and Khalatnikov, I.M., "Investigations in relativistic cosmology", Adv. Phys., 12, 185-249, (1963). 3.2

[227] Lim, W.C., van Elst, H., Uggla, C., and Wainwright, J., "Asymptotic isotropization in inhomogeneous cosmology", Phys. Rev. D, 69, 103507, (2004). 7.6, 8.2

[228] Lin, S.S., "Stability of gaseous stars in spherically symmetric motions", SIAM J. Math. Anal., 28, 539-569, (1997). 4.1

[229] Lin, X.F., and Wald, R.M., "Proof of the closed universe recollapse conjecture for general Bianchi type IX cosmologies", Phys. Rev. D, 41, 2444-2448, (1990). 7.3

[230] Lindblad, H., "Well-posedness for the linearized motion of a compressible fluid with free surface boundary", Commun. Math. Phys., 236, 281-310, (2003). 2.6

[231] Lindblad, H., "Well-posedness for the motion of an incompressible liquid with free surface boundary", (February, 2004). URL (cited on 29 March 2005):

http://arXiv.org/abs/math.AP/0402327. 2.6

[232] Lindblad, H., and Rodnianski, I., "The weak null condition for Einstein's equations.", C. R. Acad. Sci., 336, 901-906, (2003). 5.2

Living Reviews in Relativity

http://www. livingreviews . org/lrr-2005-6 
[233] Lindblad, H., and Rodnianski, I., "The global stability of the Minkowski space-time in harmonic gauge", (November, 2004). URL (cited on 29 March 2005):

http://arXiv.org/abs/math.AP/0411109. 5.2

[234] Lindblom, L., and Masood-ul Alam, A.K.M., "On the spherical symmetry of static stellar models", Commun. Math. Phys., 162, 123-145, (1994). 3.1

[235] Lions, P.-L., "Compactness in Boltzmann's equation via Fourier integral operators and applications", J. Math. Kyoto Univ., 34, 391-427, (1994). 4.2

[236] Lions, P.-L., and Perthame, B., "Propagation of moments and regularity for the 3dimensional Vlasov-Poisson system", Invent. Math., 105, 415-430, (1991). 4.2

[237] Longair, M.S., Galaxy Formation, Astronomy and Astrophysics Library, (Springer, Berlin, Germany; New York, U.S.A., 1998). 7.2

[238] Maartens, R., "Brane-World Gravity", Living Rev. Relativity, 7, lrr-2004-7, (2004). URL (cited on 22 April 2005):

http://www.livingreviews.org/lrr-2004-7. 7.5

[239] Majda, A., Compressible Fluid Flow and Systems of Conservation Laws in Several Space Variables, vol. 53 of Applied Mathematical Sciences, (Springer, Berlin, Germany; New York, U.S.A., 1984). 1

[240] Makino, T., "On spherically symmetric stellar models in general relativity", J. Math. Kyoto Univ., 38, 55-69, (1998). 3.1, 4.1

[241] Makino, T., "On the spiral structure of the (R,M) diagram for a stellar model of the TolmanOppenheimer-Volkoff equation", Funkcialaj Ekvacioj, 43, 471-489, (2000). 3.1

[242] Martín-García, J.M., and Gundlach, C., "Self-similar spherically symmetric solutions of the massless Einstein-Vlasov system", Phys. Rev. D, 65, 084026-1-18, (2002). Related online version (cited on 17 January 2002):

http://arXiv.org/abs/gr-qc/0112009. 3.3

[243] Maxwell, D., "Rough solution of the Einstein constraint equations", (May, 2004). URL (cited on 15 February 2005):

http://arXiv.org/abs/gr-qc/0405088. 2.1, 2.4

[244] Maxwell, D., "Solutions of the Einstein constraint equations with apparent horizon boundaries", Commun. Math. Phys., 253, 561-583, (2004). 2.1

[245] Misner, C.W., "Mixmaster Universe”, Phys. Rev. Lett., 22, 1071-1074, (1967). 8.1

[246] Moncrief, V., "Global Properties of Gowdy Spacetimes with $T^{3} \times R$ Topology", Ann. Phys. (N.Y.), 132, 87-107, (1981). 3.6

[247] Moncrief, V., "Neighbourhoods of Cauchy horizons in cosmological spacetimes with one Killing field", Ann. Phys. (N.Y.), 141, 83-103, (1982). 6.2

[248] Moncrief, V., and Eardley, D.M., "The global existence problem and cosmic censorship in general relativity", Gen. Relativ. Gravit., 13, 887-892, (1981). 1

[249] Moss, I., and Sahni, V., "Anisotropy in the chaotic inflationary universe", Phys. Lett. B, 178, 159-162, (1986). 7.4 
[250] Mucha, P.B., "Global existence for the Einstein-Boltzmann equation in flat RobertsonWalker spacetime", Commun. Math. Phys., 203, 107-118, (1999). 4.2

[251] Müller, V., Schmidt, H.-J., and Starobinsky, A.A., "Power-law inflation as an attractor solution for inhomogeneous cosmological models.", Class. Quantum Grav., 7, 1163-1168, (1990). 7.6

[252] Narita, M., "On the existence of global solutions for $T^{3}$-Gowdy spacetimes with stringy matter", Class. Quantum Grav., 19, 6279-6288, (2002). 3.6

[253] Narita, M., "Global existence problem in $T^{3}$-Gowdy symmetric IIB superstring cosmology", Class. Quantum Grav., 20, 4983-4994, (2003). 3.6

[254] Narita, M., "Global properties of higher-dimensional cosmological spacetimes", Class. Quantum Grav., 21, 2071-2088, (2004). 3.6, 6.2

[255] Narita, M., "On initial conditions and global existence for accelerating cosmologies from string theory", (February, 2005). URL (cited on 18 March 2005):

http://arXiv.org/abs/gr-qc/0502116. 3.6, 6.2

[256] Narita, M., Torii, T., and Maeda, K., "Asymptotic singular behavior of Gowdy spacetimes in string theory", Class. Quantum Grav., 17, 4597-4613, (2000). 6.2

[257] Newman, R.P.A.C., "On the structure of conformal singularities in classical general relativity", Proc. R. Soc. London, Ser. A, 443, 473-492, (1993). 6.1

[258] Newman, R.P.A.C., "On the structure of conformal singularities in classical general relativity. II Evolution equations and a conjecture of K. P. Tod", Proc. R. Soc. London, Ser. A, 443, 493-515, (1993). 6.1

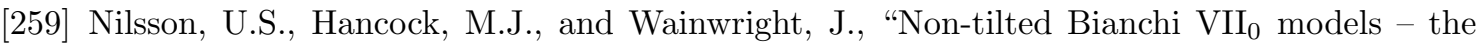
radiation fluid.", Class. Quantum Grav., 17, 3119-3134, (2000). 3.2

[260] Noundjeu, P., and Noutchegueme, N., "Local existence and continuation criterion for solutions of the spherically symmetric Einstein-Vlasov-Maxwell system", Gen. Relativ. Gravit., 36, 1373-1398, (2004). 3.3

[261] Noundjeu, P., Noutchegueme, N., and Rendall, A.D., "Existence of initial data satisfying the constraints for the spherically symmetric Einstein-Vlasov-Maxwell system", J. Math. Phys., 45, 668-676, (2004). 3.3

[262] Noutchegueme, N., Dongo, D., and Takou, E., "Global existence of solutions for the relativistic Boltzmann equation with arbitrarily large initial data on a Bianchi type I space-time", (March, 2005). URL (cited on 13 April 2005):

http://arXiv.org/abs/gr-qc/0503048. 4.2

[263] Noutchegueme, N., and Tetsadjio, M.E., "Global solutions for the relativistic Boltzmann equation in the homogeneous case on the Minkowski space-time", (July, 2003). URL (cited on 13 April 2005):

http://arXiv.org/abs/gr-qc/0307065. 4.2

[264] Olabarrieta, I., and Choptuik, M.W., "Critical phenomena at the threshold of black hole formation for collisionless matter in spherical symmetry", Phys. Rev. D, 65, 024007-1-10, (2002). 3.3

Living Reviews in Relativity

http://www.livingreviews . org/lrr-2005-6 
[265] Park, J., "Static solutions of the Einstein equations for spherically symmetric elastic bodies", Gen. Relativ. Gravit., 32, 235-252, (2000). 3.1

[266] Pfaffelmoser, K., "Global classical solutions of the Vlasov-Poisson system in three dimensions for general initial data", J. Differ. Equations, 95, 281-303, (1992). 4.2

[267] Poisson, E., and Israel, W., "Internal structure of black holes", Phys. Rev. D, 41, 1796-1809, (1990). 3.4

[268] Price, R., "Nonspherical perturbations of relativistic gravitational collapse. I. Scalar and gravitational perturbations.", Phys. Rev. D, 5, 2419-2438, (1972). 3.4

[269] Racke, R., Lectures on Nonlinear Evolution Equations: Initial Value Problems, vol. 19 of Aspects of Mathematics, (Vieweg, Wiesbaden, Germany, 1992). 1

[270] Rein, G., "Generic global solutions of the relativistic Vlasov-Maxwell system of plasma physics", Commun. Math. Phys., 135, 41-78, (1990). 4.2

[271] Rein, G., "Static solutions of the spherically symmetric Vlasov-Einstein system", Math. Proc. Camb. Phil. Soc., 115, 559-570, (1994). 3.1

[272] Rein, G., "Cosmological solutions of the Vlasov-Einstein system with spherical, plane and hyperbolic symmetry", Math. Proc. Camb. Phil. Soc., 119, 739-762, (1996). 3.6, 8.2

[273] Rein, G., "Nonlinear Stability of Homogeneous Models in Newtonian Cosmology", Arch. Ration. Mech. Anal., 140, 335-351, (1997). 9.3

[274] Rein, G., "Static shells for the Vlasov-Poisson and Vlasov-Einstein systems", Indiana Univ. Math. J., 48, 335-346, (1999). 3.1, 3.3

[275] Rein, G., "Stationary and static stellar dynamical models with axial symmetry", Nonlinear Anal., 41, 313-344, (2000). 3.1

[276] Rein, G., "Non-linear stability of gaseous stars,", Arch. Ration. Mech. Anal., 168, 115-130, (2003). $\quad 4.1$

[277] Rein, G., "On future geodesic completeness for the Einstein-Vlasov system with hyperbolic symmetry", Math. Proc. Camb. Phil. Soc., 137, 237-244, (2004). 7.2

[278] Rein, G., and Rendall, A.D., "Global existence of solutions of the spherically symmetric Vlasov-Einstein system with small initial data", Commun. Math. Phys., 150, 561-583, (1992). $\quad 3.3$

[279] Rein, G., and Rendall, A.D., "Smooth static solutions of the spherically symmetric VlasovEinstein system", Ann. Inst. Henri Poincare A, 59, 383-397, (1993). 3.1

[280] Rein, G., and Rendall, A.D., "Global Existence of Classical Solutions to the Vlasov-Poisson System in a Three Dimensional, Cosmological Setting", Arch. Ration. Mech. Anal., 126, 183-201, (1994). 4.2, 9.3

[281] Rein, G., and Rendall, A.D., "Compact support of spherically symmetric equilibria in relativistic and non-relativistic galactic dynamics", Math. Proc. Camb. Phil. Soc., 128, 363-380, (2000). $\quad 3.1$

[282] Rein, G., Rendall, A.D., and Schaeffer, J., "A regularity theorem for solutions of the spherically symmetric Vlasov-Einstein system", Commun. Math. Phys., 168, 467-478, (1995). 3.3 
[283] Rein, G., Rendall, A.D., and Schaeffer, J., "Critical collapse of collisionless matter: A numerical investigation", Phys. Rev. D, 58, 044007-1-8, (1998). 3.3

[284] Rendall, A.D., "Reduction of the characteristic initial value problem to the Cauchy problem and its applications to the Einstein equations", Proc. R. Soc. London, Ser. A, 427, 221-239, (1990). $\quad 9.4$

[285] Rendall, A.D., "The initial value problem for a class of general relativistic fluid bodies", J. Math. Phys., 33, 1047-1053, (1992). 2.6

[286] Rendall, A.D., "On the definition of post-Newtonian approximations", Proc. R. Soc. London, Ser. A, 438, 341-360, (1992). 9.2

[287] Rendall, A.D., "Cosmic censorship for some spatially homogeneous cosmological models", Ann. Phys. (N.Y.), 233, 82-96, (1994). 7.3

[288] Rendall, A.D., "The Newtonian limit for asymptotically flat solutions of the Vlasov-Einstein system", Commun. Math. Phys., 163, 89-112, (1994). 9.2

[289] Rendall, A.D., "Crushing singularities in spacetimes with spherical, plane and hyperbolic symmetry", Class. Quantum Grav., 12, 1517-1533, (1995). 3.6

[290] Rendall, A.D., "Global properties of locally homogeneous cosmological models with matter.", Math. Proc. Camb. Phil. Soc., 118, 511-526, (1995). 7.1, 7.3

[291] Rendall, A.D., "On the nature of singularities in plane symmetric scalar field cosmologies.", Gen. Relativ. Gravit., 27, 213-221, (1995). 8.2

[292] Rendall, A.D., "Constant mean curvature foliations in cosmological spacetimes", Helv. Phys. Acta, 69, 490-500, (1996). 2.1, 3.6

[293] Rendall, A.D., "The initial singularity in solutions of the Einstein-Vlasov system of Bianchi type I", J. Math. Phys., 37, 438-451, (1996). 7.1

[294] Rendall, A.D., "Existence and non-existence results for global constant mean curvature foliations", Nonlinear Anal., 30, 3589-3598, (1997). 3.6

[295] Rendall, A.D., "Existence of Constant Mean Curvature Foliations in Spacetimes with TwoDimensional Local Symmetry", Commun. Math. Phys., 189, 145-164, (1997). 3.6

[296] Rendall, A.D., "Global dynamics of the mixmaster model", Class. Quantum Grav., 14, 23412356, (1997). 3.2

[297] Rendall, A.D., "An introduction to the Einstein-Vlasov system", in Chruściel, P.T., ed., Mathematics of Gravitation, Part I: Lorentzian Geometry and Einstein Equations, Proceedings of the Workshop on Mathematical Aspects of Theories of Gravitation, held in Warsaw, February 29 - March 30, 1996, vol. 41 of Banach Center Publications, 35-68, (Polish Academy of Sciences, Institute of Mathematics, Warsaw, Poland, 1997). 3.3

[298] Rendall, A.D., "Solutions of the Einstein equations with matter", in Francaviglia, M., Longhi, G., Lusanna, L., and Sorace, E., eds., General Relativity and Gravitation, Proceedings of the 14th International Conference on General Relativity and Gravitation, Florence, Italy, 6-12 August 1995, 313-335, (World Scientific, Singapore; River Edge, U.S.A., 1997). 3.1

Living Reviews in Relativity

http: //www . livingreviews . org/lrr-2005-6 
[299] Rendall, A.D., "Blow-up for solutions of hyperbolic PDE and spacetime singularities", in Depauw, N., Robert, D., and Saint-Raymond, X., eds., Proceedings of Journées Equations aux Dérivées Partielles, La Chapelle sur Erdre, Nantes, France, 5-9 June 2000, XIV-1-12, (University of Nantes, Nantes, France, 2000). Related online version (cited on 1 March 2002): http://www. numdam.org/item?id=JEDP_2000____A14_0. 6.2

[300] Rendall, A.D., "Fuchsian analysis of singularities in Gowdy spacetimes beyond analyticity", Class. Quantum Grav., 17, 3305-3316, (2000). 6.2

[301] Rendall, A.D., "Collection of equations", personal homepage, Max Planck Institute for Gravitational Physics, (2002). URL (cited on 30 March 2005):

http://www.aei.mpg.de/〜rendall/3+1.ps. 1

[302] Rendall, A.D., "Cosmological Models and Centre Manifold Theory", Gen. Relativ. Gravit., 34, 1277-1294, (2002). Related online version (cited on 21 January 2002): http://arXiv.org/abs/gr-qc/0112040. 3.2, 7.4

[303] Rendall, A.D., "Accelerated cosmological expansion due to a scalar field whose potential has a positive lower bound", Class. Quantum Grav., 21, 2445-2454, (2004). 7.3, 7.4, 7.4

[304] Rendall, A.D., "Asymptotics of solutions of the Einstein equations with positive cosmological constant.", Ann. Henri Poincare, 5, 1041-1064, (2004). 5.1, 6.3, 7.6

[305] Rendall, A.D., "Mathematical properties of cosmological models with accelerated expansion", (August, 2004). URL (cited on 30 March 2005): http://arXiv.org/abs/gr-qc/0408053. 7, 7.5

[306] Rendall, A.D., "Intermediate inflation and the slow-roll approximation", Class. Quantum Grav., 22, 1655-1666, (2005). 7.4

[307] Rendall, A.D., and Schmidt, B.G., "Existence and properties of spherically symmetric static fluid bodies with given equation of state", Class. Quantum Grav., 8, 985-1000, (1991). 3.1

[308] Rendall, A.D., and Tod, K.P., "Dynamics of spatially homogeneous solutions of the EinsteinVlasov equations which are locally rotationally symmetric", Class. Quantum Grav., 16, 17051726, (1999). 3.2

[309] Rendall, A.D., and Uggla, C., "Dynamics of spatially homogeneous locally rotationally symmetric solutions of the Einstein-Vlasov equations", Class. Quantum Grav., 17, 4697-4713, (2000). $\quad 3.2$

[310] Rendall, A.D., and Weaver, M., "Manufacture of Gowdy spacetimes with spikes", Class. Quantum Grav., 18, 2959-2975, (2001). 8.3, 8.4

[311] Ringström, H., "The Bianchi IX attractor", Ann. Henri Poincare, 2, 405-500, (2000). 3.2, 8.1

[312] Ringström, H., "Curvature blow up in Bianchi VIII and IX vacuum spacetimes", Class. Quantum Grav., 17, 713-731, (2000). 3.2, 8.1

[313] Ringström, H., "The future asymptotics of Bianchi VIII vacuum solutions", Class. Quantum Grav., 18, 3791-3824, (2001). 7.1

[314] Ringström, H., "Future asymptotic expansions of Bianchi VIII vacuum metrics", Class. Quantum Grav., 20, 1943-1990, (2003). 7.1 
[315] Ringström, H., "Asymptotic expansions close to the singularity in Gowdy spacetimes", Class. Quantum Grav., 21, S305-S322, (2004). 8.2

[316] Ringström, H., "On a wave map equation arising in general relativity", Commun. Pure Appl. Math., 57, 657-703, (2004). 3.6, 7.2, 8.4

[317] Ringström, H., "On Gowdy vacuum spacetimes", Math. Proc. Camb. Phil. Soc., 136, 485$512,(2004) . \quad 8.2$

[318] Schaeffer, J., "A class of counterexamples to Jeans' theorem for the Vlasov-Einstein system", Commun. Math. Phys., 204, 313-327, (1999). 3.1

[319] Secchi, P., "On the equations of viscous gaseous stars", Ann. Scuola Norm. Sup. Pisa, 18, 295-318, (1991). 2.6

[320] Shapiro, S.L., and Teukolsky, S.A., "Relativistic stellar dynamics on the computer. II. Physical applications", Astrophys. J., 298, 58-79, (1985). 3.1

[321] Shapiro, S.L., and Teukolsky, S.A., "Scalar gravitation - a laboratory for numerical relativity", Phys. Rev. D, 47, 1529-1540, (1993). 4.2

[322] Sideris, T., "Formation of singularities in three-dimensional compressible fluids", Commun. Math. Phys., 101, 475-485, (1979). 4.1

[323] Smoller, J.A., and Temple, B., "Global solutions of the relativistic Euler equations", Commun. Math. Phys., 156, 65-100, (1993). 4.1

[324] Smoller, J.A., Wasserman, A.G., Yau, S.-T., and McLeod, J.B., "Smooth static solutions of the Einstein-Yang-Mills equations", Commun. Math. Phys., 143, 115-147, (1991). 3.1

[325] Ståhl, F., "Fuchsian analysis of $S^{2} \times S^{1}$ and $S^{3}$ Gowdy models", Class. Quantum Grav., 19, 4483-4504, (2002). Related online version (cited on 31 January 2002): http://arXiv.org/abs/gr-qc/0109011. 6.2

[326] Starobinsky, A.A., "Isotropization of arbitrary cosmological expansion given an effective cosmological constant", J. Exp. Theor. Phys. Lett., 37, 66-69, (1983). 6.3, 7.6

[327] Strauss, W., Nonlinear Wave Equations, vol. 73 of Regional Conference Series in Mathematics, (American Mathematical Society, Providence, U.S.A., 1989). 1

[328] Struwe, M., "Equivariant wave maps in two space dimensions.", Commun. Pure Appl. Math., $\mathbf{5 6}, 815-823,(2003) . \quad 3.3$

[329] Stuart, D.M.A., "Geodesics and the Einstein nonlinear wave system", J. Math. Pures Appl., 83, 541-587, (2004). 9.6

[330] Tanimoto, M., "Linear perturbations of spatially locally homogeneous spacetimes", in Duggal, K.L., and Sharma, R., eds., Recent Advances in Riemannian and Lorentzian Geometries, vol. 337 of Contemporary Mathematics, 171-185, (American Mathematical Society, Providence, U.S.A., 2003). 7.2

[331] Tanimoto, M., "Harmonic analysis of linear fields on the nilgeometric cosmological model", J. Math. Phys., 45, 4896-4919, (2004). 7.2

[332] Tanimoto, M., "Scalar fields on $S L(2, R)$ and $H^{2} \times R$ geometric spacetimes and linear perturbations", Class. Quantum Grav., 21, 5355-5374, (2004). 7.2

Living Reviews in Relativity

http: //www . livingreviews . org/lrr-2005-6 
[333] Tao, T., "Geometric renormalization of large energy wave maps", (November, 2004). URL (cited on 30 March 2005): http://arXiv.org/abs/math.AP/0411354. 2.4

[334] Taylor, M.E., Pseudodifferential Operators and Nonlinear PDE, vol. 100 of Progress in Mathematics, (Birkhäuser, Boston, U.S.A., 1991). 2.3

[335] Taylor, M.E., Partial Differential Equations, 3 vols., Applied Mathematical Sciences, (Springer, Berlin, Germany; New York, U.S.A., 1996). 1

[336] Tchapnda, S.B., "Structure of solutions near the initial singularity for the surface-symmetric Einstein-Vlasov system", Class. Quantum Grav., 21, 5333-5346, (2004). 3.6, 8.2

[337] Tchapnda, S.B., and Noutchegueme, N., "The surface-symmetric Einstein-Vlasov system with cosmological constant.", (April, 2003). URL (cited on 17 April 2005):

http://arXiv.org/abs/gr-qc/0304098. 3.6, 7.6

[338] Tchapnda, S.B., and Rendall, A.D., "Global existence and asymptotic behaviour in the future for the Einstein-Vlasov system with positive cosmological constant", Class. Quantum Grav., 20, 3037-3049, (2003). 3.6, 7.6

[339] Tegankong, D., "Global existence and future asymptotic behaviour for solutions of the Einstein-Vlasov-scalar field system with surface symmetry", (January, 2005). URL (cited on 17 March 2005):

http://arXiv.org/abs/gr-qc/0501062. 7.2

[340] Tegankong, D., Noutchegueme, N., and Rendall, A.D., "Local existence and continuation criteria for solutions of the Einstein-Vlasov-scalar field system with surface symmetry", $J$. Hyperbol. Differ. Equations, 1, 691-724, (2004). 7.6

[341] Thurston, W., Three-dimensional geometry and topology, Vol. 1, vol. 35 of Princeton Mathematical Series, (Princeton University Press, Princeton, U.S.A., 1997). 7.2

[342] Tod, K.P., "Isotropic cosmological singularities: other matter models", Class. Quantum Grav., 20, 521-534, (2003). 6.1

[343] van der Bij, J.J., and Radu, E., "On rotating regular nonabelian solitons", Int. J. Mod. Phys. A, 17, 1477-1490, (2002). 3.1

[344] Wainwright, J., and Ellis, G.F.R., Dynamical Systems in Cosmology, (Cambridge University Press, Cambridge, U.K.; New York, U.S.A., 1997). 3.2, 7.1

[345] Wainwright, J., Hancock, M.J., and Uggla, C., "Asymptotic self-similarity breaking at late times in cosmology", Class. Quantum Grav., 16, 2577-2598, (1999). 3.2

[346] Wald, R.M., "Asymptotic behaviour of homogeneous cosmological models with cosmological constant", Phys. Rev. D, 28, 2118-2120, (1983). 7.3

[347] Weaver, M., "Dynamics of magnetic Bianchi $\mathrm{VI}_{0}$ cosmologies.", Class. Quantum Grav., 17, $421-434,(2000) . \quad 3.2$

[348] Weaver, M., "On the area of the symmetry orbits in $T^{2}$ symmetric spacetimes with Vlasov matter", Class. Quantum Grav., 21, 1079-1098, (2004). 3.6

[349] Witt, D., "Vacuum spacetimes that admit no maximal slice", Phys. Rev. Lett., 57, 13861389, (1986). 2.1 
[350] Wolansky, G., "Static Solutions of the Vlasov-Einstein System", Arch. Ration. Mech. Anal., 156, 205-230, (2001). 4.2

[351] Woodhouse, N.M.J., "Integrability and Einstein's equations", in Chruściel, P.T., ed., Mathematics of Gravitation, Part I: Lorentzian Geometry and Einstein Equations, Proceedings of the Workshop on Mathematical Aspects of Theories of Gravitation, held in Warsaw, Poland, February 29 - March 30, 1996, vol. 41 of Banach Center Publications, 221-232, (Polish Academy of Sciences, Institute of Mathematics, Warsaw, Poland, 1997). 3.5

[352] Wu, S., "Well-posedness in Sobolev spaces of the full water wave problem in 3-D", J. Amer. Math. Soc., 12, 445-495, (1999). 2.6

[353] York Jr, J.W., "Conformal "Thin-Sandwich" Data for the Initial-Value Problem of General Relativity", Phys. Rev. Lett., 82, 1350-1353, (1999). 2.1

[354] Zipser, N., The global nonlinear stability of the trivial solution of the Einstein-Maxwell equations, Ph.D. Thesis, (Harvard University, Cambrige, U.S.A., 2000). 5.2 